Tom 26 № 4

\title{
ЗАДАЧА СТЕЧКИНА О НАИЛУЧШЕМ ПРИБЛИЖЕНИИ НЕОГРАНИЧЕННОГО ОПЕРАТОРА ОГРАНИЧЕННЫМИ И РОДСТВЕННЫЕ ЕЙ ЗАДАЧИ ${ }^{1}$
}

\author{
В. В. Арестов, Р. Р. Акопян
}

\author{
Посвящается памяти \\ Сергея Борисовича Стечкина \\ к 100-летию со дня рождения
}

\begin{abstract}
В данной статье обсуждаются задача Стечкина о наилучшем приближении линейного неограниченного оператора линейными ограниченными операторами и родственные ей экстремальные задачи. Наибольшее внимание уделено приближению операторов дифференцирования в пространствах Лебега на оси и оператору продолжения аналитической функции в область с части границы области. Это обзорная статья; она написана по материалам доклада авторов 14 сентября 2020 г. на Х Интернетвидеоконференции "День математика и механика" четырех институтов РАН: Институт математики и механики им. Н. Н. Красовского УрО РАН (г. Екатеринбург), Институт математики им. С. Л. Соболева СО PAН (г. Новосибирск), Математический институт им. В. А. Стеклова (г. Москва), Санкт-Петербургское отделение Математического института им. В.А. Стеклова. Сообщение авторов было посвящено 100-летию со дня рождения С. Б. Стечкина. Задача о наилучшем приближении линейного неограниченного оператора ограниченными - одна из составляющих его наследия. Мы старались хотя бы частично отразить появившиеся в этой тематике новые результаты, методы и новые постановки после выхода обзорных статей (Арестов, Габушин, 1995-1996). По этой тематике материала очень много, и его отбор для доклада и статьи - ответственность авторов.
\end{abstract}

Ключевые слова: задача Стечкина, восстановление, неограниченный линейный оператор, оператор дифференцирования, неравенство Колмогорова, аналитические функции, граничные значения.

V. V. Arestov, R. R. Akopyan. Stechkin's problem on the best approximation of an unbounded operator by bounded ones and related problems.

This paper discusses Stechkin's problem on the best approximation of a linear unbounded operator by bounded linear operators and related extremal problems. The main attention is paid to the approximation of differentiation operators in Lebesgue spaces on the axis and to the operator of the continuation of an analytic function to a domain from a part of the boundary of the domain. This is a review paper based on the materials of the authors' lecture on September 14, 2020, at the X Internet video-conference "Day of Mathematics and Mechanics" of four institutes of the Russian Academy of Sciences: Krasovskii Institute of Mathematics and Mechanics of the Ural Branch of RAS (Yekaterinburg), Sobolev Institute of the Siberian Branch of RAS (Novosibirsk), Steklov Mathematical Institute (Moscow), and the St. Petersburg Department of the Steklov Mathematical Institute. The lecture of the authors was dedicated to the 100th anniversary of the birth of Sergei Borisovich Stechkin. The problem of the best approximation of a linear unbounded operator by bounded ones is one of his legacies. We tried to at least partially reflect the new results, methods, and statements that appeared in this topic after the publication of the review papers (Arestov, Gabushin, 1995-1996). The material on this topic is wide; the selection of the material for the lecture and paper is the responsibility of the authors.

Keywords: Stechkin's problem, recovery, unbounded linear operator, differentiation operator, Kolmogorov inequality, analytic functions, boundary values.

MSC: 26D10, 47A58

DOI: $10.21538 / 0134-4889-2020-26-4-7-31$

\footnotetext{
${ }^{1}$ Работа выполнена в рамках исследований, проводимых в Уральском математическом центре, при поддержке РФФИ (проект 18-01-00336) и Программы повышения конкурентоспособности УрФУ (постановление № 211 Правительства РФ от 16.03.2013, контракт № 02.А03.21.0006 от 27.08.2013).
} 


\section{Введение}

Задача о наилучшем приближении неограниченного линейного оператора линейными ограниченными операторами на классе элементов банахова пространства занимает важное место в исследованиях С.Б. Стечкина, его учеников и ряда других математиков. Впервые она появилась (неявно) в статье С. Б. Стечкина [1] 1965 года; его работа [2] 1967 года содержала постановку задачи, общие принципиальные результаты и решение задачи для операторов дифференцирования малого порядка в равномерной норме на прямой и полупрямой; обе статьи можно найти в [3].

Задача Стечкина тесно связана с теорией некорректных задач, а именно, с задачей об оптимальной регуляризации вычисления значений неограниченного оператора на элементах класса (класса корректности), заданных с известной ошибкой (погрешностью), см., в частности, [4-6]. Важным стимулом ее исследования явилась ее связь с точными неравенствами между нормами производных дифференцируемых функций (неравенствами Колмогорова), см. обзорные статьи [5-7], монографию [8] и приведенную в них библиографию. Впрочем, эти вопросы будут обсуждаться в данной статье ниже.

За прошедшее время выяснена связь задачи Стечкина с несколькими экстремальными задачами. Установлены соотношения (в ряде случаев двойственные) между модулем непрерывности линейного неограниченного оператора на классе элементов пространства, наилучшим приближением такого оператора линейными ограниченными операторами и некорректной задачей оптимального восстановления значений оператора на элементах класса в предположении, что эти элементы заданы неточно (с известной погрешностью), а также между первыми двумя задачами и приближением одного класса элементов другим. Дано решение задачи для конкретных операторов в классических функциональных пространствах. При этом наиболее полно исследовано наилучшее приближение операторов дифференцирования порядка $k$ на классе $n$ раз дифференцируемых функций $(0 \leq k<n)$ в пространствах $L_{\gamma}=L_{\gamma}(I)$ на числовой оси $I=(-\infty, \infty)$ и полуоси $I=[0, \infty)$. На этом пути найдены наилучшие константы в неравенствах Колмогорова в ряде новых случаев. Точные формулировки результатов и дальнейшие ссылки можно найти в [5;6]. Для функций многих переменных перечисленные задачи исследованы существенно хуже, однако, и здесь имеется ряд точных, интересных результатов, см. разд. 6 ниже.

В последние несколько десятков лет все эти задачи интенсивно изучаются еще в одном разделе теории функций: оптимальное восстановление аналитических в области функций по их приближенно заданным значениям на части границы области; соответствующая задача Стечкина о наилучшем приближении оператора, который ставит значениям функции на части границы ее значения на подмножестве области; модуль непрерывности такого оператора. Этому будет посвящена последняя часть нашей статьи.

В данной статье обсуждаются задача Стечкина о наилучшем приближении линейного неограниченного оператора линейными ограниченными операторами и родственные ей экстремальные задачи. Наибольшее внимание уделено приближению операторов дифференцирования в пространствах Лебега на оси и оператору продолжения аналитической функции в область с части границы области. Эта обзорная статья написана по материалам доклада авторов 14 сентября 2020 года на Х Интернет-видеоконференции "День математика и механика" четыpeх институтов РАН: Институт математики и механики им. Н. Н. Красовского УрО РАН, Институт математики им. С. Л. Соболева СО РАН, Математический институт им. В. А. Стеклова, Санкт-Петербургское отделение Математического института им. В.А. Стеклова. Сообщение авторов было посвящено 100-летию со дня рождения С. Б. Стечкина. Задача о наилучшем приближении линейного неограниченного оператора ограниченными - одна из составляющих его наследия. Мы старались хотя бы частично отразить появившиеся в этой тематике новые результаты, методы и новые постановки после выхода обзорных статей [5;6]. По этой тематике материала очень много, и его отбор для доклада и статьи - ответственность авторов. 


\section{1. Задача Стечкина о наилучшем приближении неограниченного линейного оператора линейными ограниченными операторами на некотором классе элементов}

\section{1. Постановка задачи Стечкина}

Введем следующие обозначения: $X, Y$ - два банаховых пространства; $A$ - линейный неограниченный оператор из $X$ в $Y ; \mathcal{D}(A)$ - область определения оператора $A ; Q \subset \mathcal{D}(A)-$ класс элементов из $\mathcal{D}(A) ; \mathcal{B}=\mathcal{B}(X, Y)$ - множество всех линейных ограниченных операторов $T$ из $X$ в $Y ; \mathcal{B}(N)=\mathcal{B}(X, Y ; N)=\left\{T \in \mathcal{B}(X, Y):\|T\|_{X \rightarrow Y} \leq N\right\}, N>0$.

Для оператора $T \in \mathcal{B}(X, Y)$

$$
U(T)=\sup \left\{\|A x-T x\|_{Y}: x \in Q\right\}
$$

есть уклонение оператора $T$ от оператора $A$ на классе $Q$; величина же

$$
E(N)=\inf \{U(T): T \in \mathcal{B}(X, Y ; N)\}
$$

есть наилучшее приближение оператора $A$ на классе $Q$ линейными ограниченными операторами (с нормой, не превосходящей числа $N$ ).

Задача Стечкина состоит в исследовании следующих вопросов: условия конечности величины (1.1), порядок поведения по $N$ при $N \rightarrow \infty$, оценки, точное значение; существование, единственность экстремального оператора, на котором достигается нижняя грань в (1.1); связь задачи с другими экстремальными задачами.

\section{2. Оценка Стечкина величины наилучшего приближения оператора через его модуль непрерывности}

Каждую из двух функций переменного $\delta \in[0, \infty)$

$$
\begin{gathered}
\Omega(\delta)=\sup \left\{\left\|A x_{1}-A x_{2}\right\|_{Y}: x_{1}, x_{2} \in Q,\left\|x_{1}-x_{2}\right\|_{X} \leq \delta\right\} \\
\omega(\delta)=\sup \left\{\|A x\|_{Y}: x \in Q,\|x\|_{X} \leq \delta\right\}
\end{gathered}
$$

называют модулем непрерывности оператора $A$ на классе $Q$. Если класс $Q$ есть центрально симметричное и выпуклое множество, то эти характеристики связаны формулой [4]

$$
\Omega(2 \delta)=2 \omega(\delta), \quad \delta \geq 0 .
$$

Следующая теорема С. Б. Стечкина [2] дает простую, но часто используемую и эффективную оценку снизу величины наилучшего приближения (1.1) оператора через его модуль непрерывности (1.3). Положим

$$
\begin{gathered}
R(N)=R(N, Q)=\sup \{\omega(\delta)-N \delta: \delta \geq 0\}=\sup \left\{\|A x\|_{Y}-N\|x\|_{X}: x \in Q\right\} \\
\ell(\delta)=\inf \{E(N)+N \delta: N \geq 0\} .
\end{gathered}
$$

Теорема 1. Если $A-$-днородный (в частности, линейный) оператор, $Q-$ центрально симметричное выпуклое множество из области определения оператора $A$, то имеют место неравенства

$$
\begin{gathered}
E(N) \geq R(N), \quad N \geq 0 \\
\omega(\delta) \leq \ell(\delta), \quad \delta \geq 0 .
\end{gathered}
$$


Вопрос о том, при каких условиях неравенства (1.4), (1.5) обращаются в равенства, является важным и до сих пор нерешенным; наличие равенства в (1.4), (1.5) существенно облегчает решение обеих задач: задачи Стечкина (1.1) и вычисления модуля непрерывности оператоpa (1.3). Известно [9], что для функционалов оба эти неравенства обращаются в равенство. В общем случае равенства в (1.4), (1.5) не имеют места.

Может случиться, что при любом $\delta>0$ величина $\omega(\delta)$ конечна, а величина $E(N)$ не является конечной для всех $N>0$. Возможно также, что все рассматриваемые величины конечны, но неравенства (1.4), (1.5) являются строгими. Эта ситуация имеет место уже для приближения операторов дифференцирования в пространствах $L_{p}(-\infty, \infty)$ и будет обсуждаться ниже.

\section{2. Наилучшее приближение оператора дифференцирования в пространствах Лебега на оси и полуоси}

\section{1. Обозначения}

В данном разделе используются стандартные обозначения следующих классических комплексных пространств комплекснозначных, измеримых на числовой прямой функций на оси $I=(-\infty, \infty)$ и полуоси $I=[0, \infty)$. При $1 \leq \gamma<\infty$ через $L_{\gamma}=L_{\gamma}(I)$ обозначается пространство измеримых на $I$ функций $f$ таких, что функция $|f|^{\gamma}$ суммируема на оси; это пространство наделено нормой

$$
\|f\|_{\gamma}=\|f\|_{L_{\gamma}}=\left(\int|f(t)|^{\gamma} d t\right)^{1 / \gamma}
$$

здесь и ниже в интегралах по $I$ множество интегрирования не указано. Пространство $L_{1}$ часто обозначается коротко через $L$. Символами $L_{\infty}=L_{\infty}(I)$ обозначается пространство измеримых существенно ограниченных функций на оси; оно наделено нормой

$$
\|f\|_{\infty}=\|f\|_{L_{\infty}}=\operatorname{ess} \sup \{|f(t)|: t \in I\} .
$$

Это пространство содержит пространство $C=C(I)$ непрерывных ограниченных функций на оси, наделенное равномерной нормой

$$
\|f\|_{C}=\sup \{|f(t)|: t \in I\} .
$$

В свою очередь, $C=C(I)$ содержит подпространство $C_{0}=C_{0}(I)$ функций, имеющих нулевой предел на бесконечности. Для единообразия записи ниже в некоторых ситуациях под $L_{\infty}$ будет пониматься пространство $C$ или даже пространство $C_{0}$; эти ситуации будут оговариваться особо.

\section{2. Постановка задачи. Связь с неравенством Колмогорова}

$\mathrm{K}$ настоящему времени наиболее полно исследовано наилучшее приближение операторов дифференцирования $D^{k}$ порядка $k$ на классе $n$ раз дифференцируемых функций $(0 \leq k<n)$ в пространствах Лебега $L_{\gamma}=L_{\gamma}(I)$ на числовой оси $I=(-\infty, \infty)$ и полуоси $I=[0, \infty)$.

Пусть $p, q, r$ - вещественные параметры, удовлетворяющие ограничениям $1 \leq p, q, r \leq \infty$. Для целого $n \geq 1$ определим пространство $W_{r, p}^{n}=W_{r, p}^{n}(I)$ функций $f \in L_{r}$, которые $n-1$ раз непрерывно дифференцируемы на $I$, производная $f^{(n-1)}$ порядка $n-1$ локально абсолютно непрерывна, а $f^{(n)} \in L_{p}$. В пространстве $W_{r, p}^{n}$ выделим класс

$$
Q=Q_{r, p}^{n}=\left\{f \in L_{r}: f^{(n)} \in L_{p},\left\|f^{(n)}\right\|_{L_{p}} \leq 1\right\} .
$$

Рассмотрим задачу Стечкина о наилучшем приближении в пространстве $L_{q}(I)$ операторов дифференцирования $D^{k}$ порядка $k$ на классе (2.1):

$$
E(N)=E_{n, k}(N)=\inf \left\{U(T):\|T\|_{L_{r} \rightarrow L_{q}} \leq N\right\},
$$




$$
U(T)=\sup \left\{\left\|f^{(k)}-T f\right\|_{L_{q}}: f \in Q_{r, p}^{n}\right\} .
$$

Этот вариант задачи Стечкина исследовали многие математики: С. Б. Стечкин, Л. В. Тайков, Ю.Н. Субботин, В.Н. Габушин, В.И. Бердышев; В. М. Тихомиров и его ученики: А. П. Буслаев, Г. Г. Магарил-Ильяев; В.Ф. Бабенко, его коллеги и ученики; В. В. Арестов, М. А. Филатова, Е. Е. Бердышева, А. А. Кошелев и многие другие.

Модуль непрерывности операторов дифференцирования $D^{k}$ на классе $(2.1)$

$$
\omega(\delta)=\sup \left\{\left\|f^{(k)}\right\|_{L_{q}}:\|f\|_{L_{r}} \leq \delta,\left\|f^{(n)}\right\|_{L_{p}} \leq 1\right\}
$$

является однородной функцией от параметра $\delta$, а именно (за исключением некоторых, вырожденных случаев) имеет место формула (см., например, [6])

$$
\begin{gathered}
\omega(\delta)=K \delta^{\alpha}, \quad K=\omega(1), \\
\alpha=(n-k-1 / p+1 / q) /(n-1 / p+1 / r) .
\end{gathered}
$$

Нетрудно понять, что $K=\omega(1)$ есть наименьшая возможная (наилучшая) константа в неравенстве (Колмогорова) между нормами производных дифференцируемых функций

$$
\left\|f^{(k)}\right\|_{L_{q}} \leq K\|f\|_{L_{r}}^{\alpha}\left\|f^{(n)}\right\|_{L_{p}}^{1-\alpha} .
$$

Результат С. Б. Стечкина (1.4) в данном случае принимает вид

$$
E(N) \geq \beta \alpha^{\alpha / \beta} K^{1 / \beta} N^{-\alpha / \beta}, \quad \beta=1-\alpha .
$$

Неравенство (2.3) впервые появилось в работе Харди и Литтлвуда ([10], 1912); они показали, что в равномерной норме на оси (т. е. в $C(-\infty, \infty))$ при всех $k, n(1 \leq k<n)$ имеет место неравенство

$$
\left\|f^{(k)}\right\|_{C} \leq C_{n, k}\|f\|_{C}^{(n-k) / n}\|f\|_{L_{\infty}}^{k / n}, \quad f \in W_{\infty, \infty}^{n},
$$

с некоторой конечной константой. Первые точные неравенства были получены Ландау ([11], $1913)$ и Адамаром $([12], 1914)$ при $n=2, k=1$ в равномерной норме соответственно на полуоси и оси. Для $n=3,4$ при всех $1 \leq k<n$ и $n=5, k=2$ точное неравенство (2.5) на оси получил Г. Е. Шилов ([13], 1937). А. Н. Колмогоров ([14], 1939) нашел точную константу в неравенстве $(2.5)$ для всех $k, n(1 \leq k<n)$; экстремальной в этом неравенстве является известная функция Фавара - Ахиезера - Крейна (сплайн Эйлера порядка $n$ )

$$
f_{n}(t)=\frac{4}{\pi} \sum_{\ell=0}^{\infty} \frac{\sin ((2 \ell+1) t-n \pi / 2)}{(2 \ell+1)^{n+1}}, \quad t \in \mathbb{R} .
$$

Более полную информацию о случаях точного неравенства (2.3) можно найти в [6;8]. Неравенство (2.3) не всегда существует, т. е. константа $K$ в неравенстве (2.3) не всегда конечная. В. Н. Габушин $([15], 1967)$ доказал, что необходимым и достаточным условием конечности константы $K$ в (2.3) является выполнение неравенства

$$
\frac{n-k}{r}+\frac{k}{p} \geq \frac{n}{q}
$$

А. П. Буслаев, Г. Г. Магарил-Ильяев, В. М. Тихомиров ([16], 1982) доказали, что если в (2.7) выполняется строгое неравенство, то в (2.3) существует экстремальная функция. В случае, когда (2.7) обращается в равенство на оси, вероятно, "вырожденной" экстремальной функцией будет периодическая функция; при $n=2$ этот факт нетрудно обосновать. 


\section{3. Приближение операторов дифференцирования}

Первые точные результаты в задаче приближения операторов дифференцирования $(2.2)$ получил С. Б. Стечкин $[1 ; 2]$. Он нашел решение задачи $(2.2)$ в равномерной норме $(p=q=$ $r=\infty)$ на оси $I=(-\infty, \infty)$ и полуоси $I=[0, \infty)$ при $n=2, n=3,1 \leq k<n$. Он доказал, что экстремальными на оси являются классические (конечноразностные) операторы $T_{n, k}^{h}$ :

$$
\begin{gathered}
\left(T_{2,1}^{h} x\right)(t)=\left(T_{3,1}^{h} x\right)(t)=\frac{x(t+h)-x(t-h)}{2 h}, \quad N=h^{-1}, \\
\left(T_{3,2}^{h} x\right)(t)=\frac{x(t+h)-2 x(t)+x(t-h)}{h^{2}}, \quad N=\frac{4}{h^{2}} ;
\end{gathered}
$$

в случае же полуоси $I=[0, \infty)$ экстремальными являются операторы

$$
\begin{gathered}
\left(S_{2,1}^{h} x\right)(t)=\frac{x(t+h)-x(t)}{h}, \quad N=2 h^{-1}, \\
\left(S_{3,1}^{h} x\right)(t)=\frac{-8 x(t)+9 x(t+h)-x(t+3 h)}{6 h}, \quad N=\frac{3}{h}, \\
\left(S_{3,2}^{h} x\right)(t)=\frac{2 x(t)-3 x(t+h)+x(t+3 h)}{3 h^{2}}, \quad N=\frac{2}{h^{2}} .
\end{gathered}
$$

Все эти операторы являются классическими конечноразностными операторами; заслуга С. Б. Стечкина состоит в том, что он доказал их экстремальность.

При $n=4,5$ решение этого случая задачи (2.2) на оси нашел В. В. Арестов ([17], 1967), а при произвольном $n \geq 6-$ А. П. Буслаев ([18], 1981). При $n \geq 4$ экстремальные операторы бесконечноразностные с равномерными узлами.

Решение задачи Стечкина (2.2) на полуоси в равномерной норме при $n \geq 4$ на данный момент неизвестно. Впрочем, результаты и методы, приведенные в работе В. Г. Тимофеева [19], по нашему мнению, позволят выписать решение для $n=4,1 \leq k \leq 3$.

Известные авторам случаи точного решения задачи (2.2) сведены в следующую таблицу.

\begin{tabular}{cccccl}
\hline$n$ & $k$ & $q$ & $p$ & \multicolumn{1}{c}{ Авторы } \\
\hline & \multicolumn{5}{c}{$I=(-\infty, \infty)$} \\
\hline 2,3 & $1 \leq k<n$ & $\infty$ & $\infty$ & $\infty$ & С. Б. Стечкин [1;2] (1965-1967) \\
4,5 & $1 \leq k<n$ & $\infty$ & $\infty$ & $\infty$ & В. В. Арестов [17] (1967) \\
произвольное & $1 \leq k<n$ & $\infty$ & $\infty$ & $\infty$ & А. П. Буслаев [18] (1981) \\
произвольное & $1 \leq k<n$ & 2 & 2 & 2 & Ю. Н. Субботин, Л. В. Тайков [20] (1968) \\
произвольное & $0 \leq k<n$ & $\infty$ & 2 & 2 & Л. В. Тайков [21] (1968) \\
2,3 & $1 \leq k<n$ & $\infty$ & $\infty$ & $\geq 1$ & В. В. Арестов [22] (1972) \\
произвольное & $1 \leq k<n$ & 2 & 2 & 1 & В. В. Арестов [23] (1975) \\
произвольное & $1 \leq k<n$ & 1 & 1 & 1 & В. В. Арестов [24] (1969) \\
\hline & & \multicolumn{7}{c}{$I=[0, \infty)$} \\
\hline 2,3 & $1 \leq k<n$ & $\infty$ & $\infty$ & $\infty$ & С. Б. Стечкин [1;2] (1965-1967) \\
2,3 & $1 \leq k<n$ & $\infty$ & $\infty$ & $\geq 1$ & В. В. Арестов [22] (1972) \\
2 & 1 & 1 & 1 & 1 & В. И. Бердышев [25] (1971) \\
произвольное & $0 \leq k<n$ & $\infty$ & 2 & 2 & В. Н. Габушин [26] (1969) \\
2 & 1 & 2 & 2 & 2 & В. В. Арестов, М. А. Филатова [27] (2014) \\
\hline
\end{tabular}

Практически во всех перечисленных случаях для решения задачи (2.2) использовалось неравенство С. Б. Стечкина (2.4). Более того, это неравенство обратилось в равенство (в такой 
ситуации говорят, что задачи Стечкина и Колмогорова согласованы). В некоторых из этих случаев соответствующее точное неравенство (2.3) было известно ранее, в других же одновременно была найдена наименьшая константа $K$ в (2.3). При $q=p=2, r=1$ на оси задача Стечкина и неравенство Колмогорова оказались несогласованными, т. е. неравенство (2.4) является строгим, в этом случае для решения задачи Стечкина (2.2) использовались [23] соображения инвариантности задачи относительно сдвига.

Метод решения задачи и характер решения, в частности, вид экстремального оператора зависят от параметров задачи $p, q, r, n, k, I$. Опишем результат на полуоси для $n=2, k=1$, $p=q=r=2$. Соответствующее точное неравенство

$$
\left\|f^{\prime}\right\|_{L^{2}} \leq \sqrt{2\|f\|_{L^{2}}\left\|f^{\prime \prime}\right\|_{L^{2}}}, \quad f \in W_{2,2}^{2}(0, \infty)
$$

между нормами производных доказано, и даже несколькими методами, в монографии [28, гл. VII, § 7.8], вышедшей в оригинале в 1932 г.

Решение задачи Стечкина в этом случае получили М.А. Филатова и В.В. Арестов ([27], 2014). Задача Стечкина и неравенство (2.9) оказались согласованными. Экстремальный оператор строится следующим образом. Для $f \in L_{2}(0, \infty)$ рассматривается дифференциальная задача

$$
\begin{aligned}
& y^{\prime \prime \prime}-\frac{2}{3} y^{\prime \prime}-\frac{2}{3} y^{\prime}+y=f, \\
& y \in L_{2}(0, \infty), \quad y^{\prime \prime}(0)=0 .
\end{aligned}
$$

Для любой функции $f \in L_{2}(0, \infty)$ эта задача имеет единственное решение $y \in L_{2}(0, \infty)$. Аппроксимирующий оператор $T$ :

$$
T f=y^{\prime}-\frac{1}{3} y^{\prime \prime}-\frac{1}{3} y^{\prime \prime \prime}, \quad f \in L_{2}(0, \infty)
$$

Отметим без обсуждения еще результат В. И. Бердышева [25] на полуоси в пространстве $L(0, \infty)$, т. е. при $p=q=r=1$ для $n=2, k=1$. Вид экстремального оператора и обоснование его экстремальности весьма оригинальные.

Величина $E_{n, k}(N)$ не всегда конечна. Необходимое и достаточное условие ее конечности получил В. Н. Габушин $([29], 1972)$. Это условие состоит в том, что

$$
q \geq r, \quad q \geq p .
$$

Условия $(2.10),(2.7)$ конечности величины $E_{n, k}(N), N>0$, и константы $K$ в неравенстве Колмогорова (2.3) различны. Важным и до сих пор нерешенным является вопрос о том, когда (при выполнении условия (2.10), обеспечивающего конечность обеих величин $E(N)$ и $K$ ) неравенство (2.4) обращается в равенство, т. е. задачи Стечкина и Колмогорова согласованы. В большинстве случаев для конкретных значений параметров, при которых была исследована задача (2.2), задачи Стечкина и Колмогорова оказались согласованными. В следующей теореме В.В. Арестова ([30], 1992) приведены значения параметров, при которых задачи заведомо не согласованы.

Теорема 2. В следующих трех случаях:

1) $q=r=2, \quad 1 \leq p<2, \quad 0<k<n$,

2) $q=p=2, \quad 1 \leq r<2, \quad 0 \leq k<n$,

3) $r=1, \quad 1 \leq q<\infty, \quad 1<p \leq \infty, \quad 0 \leq k<n$,

неравенство (2.4) является строгим. 


\section{3. Связь задачи Стечкина с некорректной задачей восстановления значений неограниченного оператора}

Некоторые задачи вычислительной математики, теории функций, других разделов математики являются некорректными задачами восстановления значений оператора $A$ на элементах класса $Q$, лежащего в области определения $D(A)$ оператора $A$, в предположении, что элементы класса $Q$ заданы с известной погрешностью; восстановление осуществляется с помощью некоторого множества $\mathcal{R}$ операторов (однозначных отображений) пространства $X$ в $Y$. В качестве $\mathcal{R}$, как правило, берется одно из следующих множеств отображений: множество $\mathcal{O}=\mathcal{O}(X, Y)$ всех отображений пространства $X$ в пространство $Y$, множество $\mathcal{L}=\mathcal{L}(X, Y)$ линейных операторов из $X$ в $Y$, множество $\mathcal{B}=\mathcal{B}(X, Y)$ линейных ограниченных операторов из $X$ в $Y$. В дальнейшем через $\delta$ будем обозначать неотрицательный параметр, характеризующий погрешность в задании элементов класса $Q$. Для числа $\delta \geq 0$ и оператора $T \in \mathcal{R}$ полагаем

$$
U_{\delta}(T)=U_{\delta}(T ; A, Q)=\sup \left\{\|A x-T \eta\|_{Y}: x \in Q, \eta \in X,\|x-\eta\|_{X} \leq \delta\right\} .
$$

Тогда

$$
\mathcal{E}_{\delta}(\mathcal{R})=\mathcal{E}_{\delta}(\mathcal{R} ; A, Q)=\inf \left\{U_{\delta}(T): T \in \mathcal{R}\right\}
$$

есть величина наилучшего восстановления (наименьшая погрешность восстановления) оператора $A$ с помощью множества отображений (методов восстановления) $\mathcal{R}$ на элементах класса $Q$, заданных с известной погрешностью $\delta$.

Оценку снизу величин (3.1) и (3.2) дает модуль непрерывности (1.2), (1.3) оператора $A$ на классе $Q$. А именно, довольно давно известно, что

$$
\omega(\delta) \leq \mathcal{E}_{\delta}(\mathcal{O}) \leq 2 \omega(\delta) .
$$

Следующее утверждение в той или иной степени общности приводилось, начиная с 1965 г., в ряде сообщений С. Б. Стечкина на научных семинарах и конференциях; доказательство можно найти, например, в [31]. Это утверждение показывает, что не только задачи (3.2), (1.3), но и задача (1.1) взаимосвязаны.

Теорема 3. Если $A$ - однородный оператор, $Q$ - чентрально симметричное выпуклое множество, то при любом $\delta>0$

$$
\begin{gathered}
\omega(\delta) \leq \mathcal{E}_{\delta}(\mathcal{O}) \leq \mathcal{E}_{\delta}(\mathcal{B})=\mathcal{E}_{\delta}(\mathcal{L}) \leq \ell(\delta), \\
\ell(\delta)=\inf \{E(N)+N \delta: N \geq 0\}
\end{gathered}
$$

Для линейных функционалов все неравенства (3.3) обращаются в равенства [9;32].

\section{4. Приближение инвариантных операторов}

\section{1. Некоторые дополнительные обозначения}

Обозначим через $V=V(-\infty, \infty)$ пространство (комплексных) ограниченных борелевских мер на оси $(-\infty, \infty)$. Это пространство можно отождествлять с множеством (комплексных) функций $\mu$ ограниченной вариации на $(-\infty, \infty)$, значения которых в точках разрыва заключены между пределами справа и слева, а точнее, значения вещественной и мнимой частей которых в точках разрыва заключены между пределами справа и слева. Норма в пространстве $V$ есть полная вариация $\bigvee \mu=\bigvee_{-\infty}^{\infty} \mu$ меры (функции) $\mu \in V$.

Рассматриваемые нами пространства функций $\left(L^{\gamma}, C, C_{0}, V\right)$ на оси и их нормы инвариантны относительно группы сдвигов $\left\{\tau_{h}: h \in \mathbb{R}\right\}$, определенных формулой $\left(\tau_{h} f\right)(t)=f(t-h)$, 
$t \in \mathbb{R}$, и родственного семейства операторов $\left\{\sigma_{h}: h \in \mathbb{R}\right\}$, заданных формулой $\left(\sigma_{h} f\right)(t)=$ $f(h-t), t \in \mathbb{R}$. Операторы этих двух семейств связаны соотношением $\sigma_{h}=\tau_{h} \sigma_{0}$, где $\sigma_{0}-$ оператор смены знака аргумента функции: $\left(\sigma_{0} f\right)(t)=f(-t), t \in \mathbb{R}$.

Прямое и обратное преобразования Фурье функций, по крайней мере, из пространства $L$ определим соответственно формулами

$$
\widehat{f}(t)=\int e^{-2 \pi t \eta i} f(\eta) d \eta ; \quad \check{f}(t)=\int e^{2 \pi t \eta i} f(\eta) d \eta .
$$

Свойства преобразования Фурье (и его обратного) можно найти в [33, гл. I, $\S \S 1,2]$.

Пусть $\mathscr{S}$ есть (топологическое векторное) пространство бесконечно дифференцируемых функций на $\mathbb{R}$, быстро убывающих на бесконечности вместе со всеми своими производными, а $\mathscr{S}^{\prime}$ - соответствующее двойственное пространство обобщенных функций (см., например, $\left[33\right.$, гл. I, §3]). Значение функционала $\theta \in \mathscr{S}^{\prime}$ на функции $\phi \in \mathscr{S}$ будем обозначать $\langle\theta, \phi\rangle$.

\section{2. Инвариантность относительно сдвига задачи Стечкина}

Если оператор $A$ и класс $Q$ инвариантны относительно некоторого семейства преобразований, то (при определенных дополнительных условиях) в (1.1) и (3.2) можно ограничиться инвариантными аппроксимирующими операторами $T$, что в ряде случаях существенно облегчает решение задач. Такая идея известна, она применялась и ранее, в частности, в задачах приближения классов функций, инвариантных относительно сдвига, с помощью линейных методов; для обоснования применяется конструкция некоторого усреднения (см. [34, гл. 10; 35, гл. 3]).

Эти соображения можно применить в исследовании задачи Стечкина в довольно общей ситуации [36]. Но особенно полезными они оказались для изучения задачи наилучшего приближения в пространствах $L_{\gamma}\left(\mathbb{R}^{m}\right), 1 \leq \gamma \leq \infty$, неограниченных операторов, инвариантных относительно (любого) сдвига, каковыми являются, в частности, дифференциальные операторы с постоянными коэффициентами [23; 30; 36-39].

Рассмотрим более подробно наилучшее приближение оператора дифференцирования $A=$ $D^{k}=d^{k} / d t^{k}$ в пространстве $L_{q}=L_{q}(-\infty, \infty)$ на числовой прямой множеством $\mathcal{B}\left(L_{r}, L_{s} ; N\right)$ линейных ограниченных операторов из $L_{r}$ в $L_{s}$ на классе

$$
Q_{r, p}^{n}=\left\{f \in L_{r}: f^{(n)} \in L_{p},\left\|f^{(n)}\right\|_{p} \leq 1\right\} .
$$

Задача Стечкина

$$
\begin{gathered}
E(N)=E_{n, k}(N)=E_{n, k}(N ; r, s ; p, q)=\inf \left\{U(T): T \in \mathcal{B}\left(L_{r}, L_{s} ; N\right)\right\}, \\
U(T)=\sup \left\{\left\|f^{(k)}-T x\right\|_{q}: f \in Q_{r, p}^{n}\right\} .
\end{gathered}
$$

Отметим, что в (4.1) в сравнении с (2.2) не предполагается, что $s=q$.

За исключением некоторых вырожденных значений параметров можно утверждать $[23$, $\S 5]$, что $E_{n, k}(N), N>0$, конечна тогда и только тогда, когда

$$
s \geq r, \quad q \geq p,
$$

и, если $k+1 / r-1 / s>0$, то имеет место формула

$$
E_{n, k}(N)=N^{-\gamma} E_{n, k}(1), \quad \gamma=\frac{n-k+1 / q-1 / p}{k+1 / r-1 / s} .
$$

$\mathrm{K}$ тому же, в [23, теорема 1] доказано, что в задаче (4.1), по крайней мере, на множестве $Q_{r, p}^{n} \cap \mathscr{S}$ можно ограничиться множеством $\mathcal{T}\left(L^{r}, L^{s}\right)$ линейных ограниченных операторов из $L^{r}$ в $L^{s}$, инвариантных относительно сдвига. 


\section{3. Пространство мультипликаторов и ему преддуальное}

Свойства ограниченных операторов из $L_{r}$ в $L_{s}$, инвариантных относительно сдвига, можно найти, например, в монографиях [40;41]. Известно, что если $s<r$, то $\mathcal{T}\left(L_{r}, L_{s}\right)$ состоит только из нулевого оператора. При $s \geq r$ оператор $T \in \mathcal{T}\left(L_{r}, L_{s}\right)$, по крайней мере, на множестве $\mathscr{S}$ имеет вид свертки $T f=\theta * f$ с некоторым элементом $\theta=\theta_{T} \in \mathscr{S}^{\prime}$, см., например, [40, теорема 1.2]. Множество $M_{r, s}=M(r, s)$ таких обобщенных функций $\theta_{T}$ является банаховым пространством относительно нормы $\left\|\theta_{T}\right\|_{M(r, s)}=\|T\|_{L_{r} \rightarrow L_{s}}$. Пространства $M(r, s)$, называемые пространствами мультипликаторов, довольно хорошо изучены (см. монографии $[33 ; 40 ; 41])$. В частности, в некоторых случаях известно описание множества $M(r, s)$; см., например, [40, гл. I; 33, гл. 1, §3; 41, гл. 4]. Так, известно, что

$$
\begin{gathered}
M(2,2)=\widetilde{L_{\infty}}=\left\{\theta \in \mathscr{S}^{\prime}: \widehat{\theta} \in L_{\infty}\right\}, \\
M(r, \infty)=L_{r^{\prime}}, \quad 1 \leq r<\infty, \quad 1 / r+1 / r^{\prime}=1 ; \quad M(\infty, \infty)=M(1,1)=V .
\end{gathered}
$$

Утверждения (4.2) и (4.3) имеют место вместе с соответствующими равенствами для норм элементов; так $\|\theta\|_{M(2,2)}=\|\check{\theta}\|_{L_{\infty}}$ для $\theta \in M(2,2)$.

Пространство мультипликаторов $M(r, s), 1 \leq r \leq s \leq \infty$, является сопряженным для некоего функционального пространства $L(r, s)$ :

$$
(L(r, s))^{*}=M(r, s),
$$

которое естественно называть преддуальным для $M(r, s)$. Этот факт доказал А. Фига-Таламанка ([42], 1965-1967).

В. В. Арестов использует другую конструкцию преддуального пространства [43].

При $1 \leq r \leq s \leq \infty$ определим на множестве $\mathscr{S}$ функционал

$$
\|\phi\|_{r, s}=\sup \{|\langle\theta, \phi\rangle|: \theta \in M(r, s),\|\theta\| \leq 1\}, \quad \phi \in \mathscr{S},
$$

который, как нетрудно понять, является на $\mathscr{S}$ нормой. Обозначим через $L(r, s)$ пополнение $\mathscr{S}$ относительно этой нормы. Пространство $L(r, s)$ является преддуальным для $M(r, s)$.

Пространство $L(r, s)$ вложено [23;30] в $L_{\omega}, 1 / \omega=1 / r-1 / s$. Если $s=\infty$, то из (4.3) следует, что

$$
L(\infty, \infty)=C_{0}, \quad L(r, \infty)=L_{r} \quad \text { при } \quad 1 \leq r<\infty
$$

вместе с равенствами норм элементов. Помимо того, как следствие (4.2) имеем

$$
L(2,2)=\widetilde{L_{1}}=\left\{f \in C_{0}: \widehat{f} \in L_{1}\right\},
$$

причем $\|f\|_{2,2}=\|\widehat{f}\|_{L_{1}}, f \in L(2,2)$.

Как хорошо известно, имеют место вложения

$$
M(2,2) \subset M(r, r) \subset M(\infty, \infty), \quad 1 \leq r \leq \infty .
$$

Поэтому имеют место вложения

$$
L(\infty, \infty) \subset L(r, r) \subset L(2,2), \quad 1 \leq r \leq \infty .
$$

Пусть $K$ есть наилучшая константа в неравенстве

$$
\begin{gathered}
\left\|f^{(k)}\right\|_{C} \leq K\|f\|_{r, s}^{\alpha}\left\|f^{(n)}\right\|_{p, q}^{1-\alpha}, \\
\alpha=\frac{n-k+1 / q-1 / p}{n+1 / q-1 / p+1 / r-1 / s}, \quad \beta=1-\alpha .
\end{gathered}
$$

Как частный случай результата [30, теорема 3] справедливо такое утверждение. 
Теорема 4. Если $s \geq r \geq 1, q \geq p>1$, причем $s>r$ при $k=0$, то для любого значения $N>0$

$$
E_{n, k}(N)=\beta \alpha^{\alpha / \beta} K^{1 / \beta} N^{-\alpha / \beta},
$$

где $K$ - наименьшая константа в (4.5).

Таким образом, величина $E_{n, k}(N)$ выражается через наименьшую константу в неравенстве (4.5) и к тому же при $q=s$ в силу (2.4) оценивает сверху наилучшую константу в неравенстве Колмогорова (2.3).

\section{4. Частный случай}

При определенных условиях на параметры удается найти решение всех задач в (4.6). Обсудим случай [44]

$$
p=q=\infty, \quad 1 \leq s=r \leq \infty .
$$

В этом случае неравенство (4.5) имеет вид

$$
\left\|f^{(k)}\right\|_{C} \leq K_{n, k}(r)\|f\|_{r, r}^{(n-k) / n}\left(\left\|f^{(n)}\right\|_{L_{\infty}}\right)^{k / n} .
$$

При $s=r=\infty$ это есть классический вариант (2.5) неравенства (2.3) между нормами производных.

Вложения (4.4) влекут, в частности, неравенство

$$
K_{n, k}(r) \leq K_{n, k}(\infty)=C_{n, k}, \quad 1 \leq r \leq \infty
$$

где $C_{n, k}$ - константа в неравенстве (2.5).

Для нечетных $n \geq 3$ в (4.9) имеет место равенство [44].

Для четных $n \geq 2$ это, вообще говоря, уже не так. Неравенство (4.9) строгое, по крайней мере, при $n=2(k=1), r=2[45]$. В случае $r=2$ неравенство (4.8) принимает вид

$$
\left\|f^{(k)}\right\|_{C} \leq K_{n, k}(2)\|\widehat{f}\|_{L}^{(n-k) / n}\left(\left\|f^{(n)}\right\|_{L_{\infty}}\right)^{k / n}
$$

Так, при $n=2, k=1$

$$
K_{2,1}(2)=\frac{\pi}{2}\left(\frac{4}{\pi} \sum_{\ell=0}^{\infty} \frac{1}{(2 \ell+1)^{3}}\right)^{-1 / 2}<K_{2,1}(\infty)=\sqrt{2} .
$$

Что будет для четных $n \geq 4$, пока неизвестно.

Соответствующие значениям параметров (4.7) задачу Стечкина и величину наилучшего приближения (4.1) обозначим символом $E_{n, k}\left(N ; L^{r}\right)$. Как показано в [44], для нечетных $n \geq 3$ задача $E_{n, k}\left(N ; L^{r}\right)$ не зависит от $r$. В частности, экстремальные операторы, полученные С. Б. Стечкиным $(n=2,3)$, В. В. Арестовым $(n=4,5)$ и А. П. Буслаевым $(n \geq 6)$ в случае $r=\infty$, являются экстремальными при всех $r, 1 \leq r \leq \infty$.

Для четных значений $n$ ситуация иная. По крайней мере при $n=2(k=1), r=2$, в отличие от оператора (2.8), экстремальный оператор будет иметь вид свертки с сингулярным ядром [45].

\section{5. Оптимальное дифференцирование функций при известном с погрешностью преобразовании Фурье}

При $n \geq 1$ рассмотрим пространство $Y^{n}=\widehat{L} \cap W_{\infty, \infty}^{n}$ функций $f$, преобразование Фурье которых суммируемо, которые являются непрерывно дифференцируемыми $n-1$ раз на $(-\infty, \infty)$ 
и, более того, производная $f^{(n-1)}$ порядка $n-1$ которых локально абсолютно непрерывна на оси и $f^{(n)} \in L_{\infty}$. В пространстве $Y^{n}$ выделим класс $\mathcal{Q}_{n}=\left\{f \in Y^{n}:\left\|f^{(n)}\right\|_{L_{\infty}} \leq 1\right\}$.

Пусть $\mathcal{O}=\mathcal{O}(V, C)$ есть множество всех (однозначных) отображений пространства $V$ функций ограниченной вариации в пространство $C$ непрерывных ограниченных функций. Для отображения $T \in \mathcal{O}$ и параметра $\delta>0$ положим

$$
R(T)=R_{n, k}^{\delta}(T)=\sup \left\{\left\|f^{(k)}-T g\right\|_{C}: f \in \mathcal{Q}^{n}, g \in L,\|g-\widehat{f}\|_{L} \leq \delta\right\} .
$$

В данном случае априорная информация относительно функции состоит в том, что $f \in \mathcal{Q}^{n}$. Функция $f$ известна неточно. А именно, спектральная функция $\widehat{f}$ функции $f$ известна с известной погрешностью в пространстве $L=L(-\infty, \infty)$.

Наименьшее значение

$$
\varrho_{n, k}(\delta)=\inf \{R(T): T \in \mathcal{O}(V, C)\}
$$

величины (5.1) по всем отображениям $T \in \mathcal{O}$ есть наименьшая погрешность восстановления оператора дифференцирования $D^{k}=d^{k} / d t^{k}$ на функциях класса $\mathcal{Q}^{n}$, преобразование Фурье которых задано с погрешностью $\delta$ в пространстве $L$.

Задача (5.2) в определенном смысле соответствует неравенству (4.10).

В [46] приведено решение задачи (5.2) в следующих случаях: $n \geq 3$ нечетное, $n=2$; при четном $n \geq 4$ точного решения нет, даны хорошие оценки.

\section{6. Приближение операторов дифференцирования на классах функций многих переменных}

Задача Стечкина о наилучшем приближении операторов дифференцирования ограниченными операторами на классах функций многих переменных решена лишь в некоторых случаях. Здесь будут приведены наиболее интересные из них.

Пусть $W=W_{\infty}^{\left(n_{1}, n_{2}\right)}=W_{\infty}^{\left(n_{1}, n_{2}\right)}\left(\mathbb{R}^{2}\right)$ для натуральных $n_{1}, n_{2}$ есть множество вещественных функций $f(t)=f\left(t_{1}, t_{2}\right)$ двух переменных $t=\left(t_{1}, t_{2}\right)$, непрерывных и ограниченных на $\mathbb{R}^{2}$, т. е. принадлежащих пространству $C=C\left(\mathbb{R}^{2}\right)$, со следующими свойствами. Частная производная $f^{\left(n_{1}-1,0\right)}\left(t_{1}, t_{2}\right)$ функции $f \in W$ существует, локально абсолютно непрерывна по переменному $t_{1}$ на $\mathbb{R}$ почти для всех $t_{2} \in \mathbb{R}$, и производная $f^{\left(n_{1}, 0\right)}\left(t_{1}, t_{2}\right)$ существенно ограничена на $\mathbb{R}^{2}$, т. е. принадлежит пространству $L_{\infty}=L_{\infty}\left(\mathbb{R}^{2}\right)$. Аналогично, частная производная $f^{\left(0, n_{2}-1\right)}\left(t_{1}, t_{2}\right)$ функции $f \in W$ локально абсолютно непрерывна по переменному $t_{2}$ на $\mathbb{R}$ почти для всех $t_{1} \in \mathbb{R}$, и производная $f^{\left(0, n_{2}\right)}$ принадлежит пространству $L_{\infty}\left(\mathbb{R}^{2}\right)$.

Условимся в этом разделе использовать одно и то же обозначение $\|g\|$ для равномерной нормы $\|g\|_{C\left(\mathbb{R}^{2}\right)}$ функции $g \in C\left(\mathbb{R}^{2}\right)$ и $L_{\infty}$-нормы функции $g \in L_{\infty}\left(\mathbb{R}^{2}\right)$.

Известно (см., например, [47, гл. $3, \S 3.5$, п. 3.5.82]), что если целые неотрицательные числа $k_{1}, k_{2}$ удовлетворяют условию

$$
\frac{k_{1}}{n_{1}}+\frac{k_{2}}{n_{2}}<1
$$

то у функции $f \in W_{\infty}^{\left(n_{1}, n_{2}\right)}\left(\mathbb{R}^{2}\right)$ существует непрерывная, ограниченная в плоскости производная

$$
f^{\left(k_{1}, k_{2}\right)}\left(t_{1}, t_{2}\right)=\frac{\partial^{|k|} f\left(t_{1}, t_{2}\right)}{\partial t_{2}^{k_{2}} \partial t_{1}^{k_{1}}}, \quad|k|=k_{1}+k_{2} .
$$

В частности, функции $f \in W_{\infty}^{(3,3)}$ имеют непрерывные и, более того, ограниченные смешанные производные $f^{(1,1)}$.

В. Н. Коновалов доказал $([48], 1978)$, что на множестве функций $f \in W_{\infty}^{(3,3)}$ имеет место точное неравенство

$$
\left\|f^{(1,1)}\right\| \leq\left(3\|f\|\left\|f^{(3,0)}\right\|\left\|f^{(0,3)}\right\|\right)^{1 / 3}
$$


это неравенство обращается в равенство на функциях $a f_{3}\left(b_{1} t_{1}+b_{2} t_{2}\right)$, где $a, b_{1}, b_{2}$ - отличные от нуля константы, а $f_{3}-$ сплайн Эйлера третьего порядка, являющийся экстремальной функцией в неравенстве (2.5) при $n=3$ и определенный в (2.6).

Неравенству (6.1) соответствует задача

$$
\begin{gathered}
E(N)=\inf \left\{U(T):\|T\|_{C \rightarrow C} \leq N\right\}, \\
U(T)=\sup \left\{\left\|f^{(1,1)}-T f\right\|_{C}: f \in Q_{\infty}^{(3,3)}\right\},
\end{gathered}
$$

о наилучшем приближении оператора дифференцирования $D^{(1,1)}=\frac{\partial^{2}}{\partial t_{2} \partial t_{1}}$ ограниченными линейными операторами в пространстве $C=C\left(\mathbb{R}^{2}\right)$ на классе

$$
Q=Q_{\infty}^{(3,3)}=\left\{f \in W_{\infty}^{(3,3)}:\left\|f^{(3,0)}\right\| \leq 1,\left\|f^{(0,3)}\right\| \leq 1\right\} .
$$

Эту задачу решил А. П. Буслаев [18] в 1981 г. Он доказал, что

$$
E(N)=\frac{2}{3} N^{-1 / 2}
$$

и оператор

$$
\left(T_{h} f\right)\left(t_{1}, t_{2}\right)=\frac{f\left(t_{1}+h, t_{2}+h\right)-f\left(t_{1}-h, t_{2}+h\right)-f\left(t_{1}+h, t_{2}-h\right)+f\left(t_{1}-h, t_{2}-h\right)}{4 h^{2}},
$$

где $N=h^{-2}, h>0$, является экстремальным в этой задаче.

Большой интерес представляет полученное О.А.Тимошиным ([49], 1984) для функций $f \in W_{\infty}^{(3,3)}$ интегральное представление

$$
f^{(1,1)}-T_{h} f=f^{(3,0)} * G_{1}+f^{(0,3)} * G_{2},
$$

правая часть которого является суммой сверток производных $f^{(3,0)}$ и $f^{(0,3)}$ с суммируемыми на $\mathbb{R}^{2}$ функциями $G_{1}, G_{2}$, обладающими экстремальным свойством

$$
U\left(T_{h}\right)=\left\|G_{1}\right\|_{L\left(\mathbb{R}^{2}\right)}+\left\|G_{2}\right\|_{L\left(\mathbb{R}^{2}\right)} .
$$

В 1995 г. О.А. Тимошин [50] получил решение соответствующих задач при $n_{1}=3, n_{2}=2$ (или, то же самое, $n_{1}=2, n_{2}=3$ ). А именно, он доказал, что на классе функций $W^{(3,2)}$ имеет место точное неравенство

$$
\left\|f^{(1,1)}\right\| \leq\left(9\left\|f^{(3,0)}\right\|\right)^{1 / 3}\left(2\left\|f^{(0,2)}\right\|\right)^{1 / 2}\|f\|^{1 / 6} .
$$

Одновременно О.А.Тимошин решил аналогичную задачу о наилучшем приближении оператора дифференцирования $D^{(1,1)}$ ограниченными линейными операторами в пространстве $C\left(\mathbb{R}^{2}\right)$ на классе

$$
Q=Q_{\infty}^{(3,2)}=\left\{f \in W_{\infty}^{(3,2)}:\left\|f^{(3,0)}\right\| \leq 3,\left\|f^{(0,2)}\right\| \leq 2\right\} .
$$

В данном случае

$$
E(N)=5 N^{-1 / 5}
$$

О.А. Тимошин выписал также решение соответствующих некорректных задач

$$
\begin{gathered}
\mathcal{E}_{\delta}(\mathcal{R})=\mathcal{E}_{\delta}\left(\mathcal{R} ; D^{(1,1)}, Q\right)=\inf \left\{U_{\delta}\left(T ; D^{(1,1)}, Q\right): T \in \mathcal{R}\right\}, \\
U_{\delta}(T)=U_{\delta}\left(T ; D^{(1,1)}, Q\right)=\sup \left\{\left\|f^{(1,1)}-T f_{\delta}\right\|_{C}: f \in Q, f_{\delta} \in C,\left\|f-f_{\delta}\right\|_{C} \leq \delta\right\},
\end{gathered}
$$

об оптимальном вычислении значений оператора $D^{(1,1)}$ на функциях классов $Q=Q_{\infty}^{(3,3)}$ и $Q=Q_{\infty}^{(3,2)}$ в пространстве $C\left(\mathbb{R}^{2}\right)$; как и следовало ожидать, во множестве $\mathcal{O}$ всех методов 
восстановления оптимальным оказался линейный ограниченный оператор, экстремальный в задаче Стечкина при соответствующей связи параметров $\delta$ и $N$. Так для класса $Q=Q_{\infty}^{(3,3)}$ имеет место равенство

$$
\mathcal{E}_{\delta}(\mathcal{O})=\mathcal{E}_{\delta}(\mathcal{B})=(3 \delta)^{1 / 3}
$$

и (линейный ограниченный) оператор (6.2) со значением $h=(3 \delta)^{\frac{1}{3}}$ является оптимальным методом.

В. Г. Тимофеев ([51], 1985) получил для функций $f(t)=f\left(t_{1}, \ldots, t_{m}\right), m \geq 2$, переменных $t=\left(t_{1}, \ldots, t_{m}\right)$ точное неравенство

$$
\left\|\frac{\partial f}{\partial t_{i}}\right\|_{C\left(\mathbb{R}^{m}\right)} \leq \sqrt{2}\|f\|_{C\left(\mathbb{R}^{m}\right)}^{1 / 2}\|\Delta f\|_{L_{\infty}\left(\mathbb{R}^{m}\right)}^{1 / 2}, \quad i=\overline{1, m}
$$

здесь

$$
\Delta f=\frac{\partial^{2} f}{\partial t_{1}^{2}}+\cdots+\frac{\partial^{2} f}{\partial t_{m}^{2}}
$$

есть значение оператора Лапласа на функции $f$, определяемое по схеме Соболева. Неравенство (6.3) обращается в равенство на одномерных функциях $a f_{2}\left(b t_{i}\right)$, где $a, b$ - отличные от нуля константы, а $f_{2}$ - функция, определенная в (2.6).

В. Г. Тимофеевым [51] решены также соответствующая задача о наилучшем приближении в пространстве $C=C\left(\mathbb{R}^{m}\right)$ оператора дифференцирования $\frac{\partial}{\partial t_{i}}$ ограниченными линейными операторами на классе

$$
Q=\left\{f \in C\left(\mathbb{R}^{m}\right):\|\Delta f\|_{L_{\infty}\left(\mathbb{R}^{m}\right)} \leq 1\right\}
$$

и соответствующая некорректная задача оптимального восстановления значений оператора $\frac{\partial}{\partial t_{i}}$ на функциях из этого класса, заданных с погрешностью в пространстве $C$. В последних задачах экстремальный оператор является сверткой с некоторой мерой, сосредоточенной на гиперплоскостях $t_{i}= \pm h$. Как показал В. Г. Тимофеев, все эти результаты сохраняются также в пространстве $L\left(\mathbb{R}^{m}\right)$.

\section{7. Задачи для аналитических функций}

Рассматриваемый в статье круг задач на классах аналитических функций исследован гораздо меньше. Не претендуя на полноту, приведем здесь некоторые результаты.

Введем обозначения $D_{R}=\{z \in \mathbb{C}:|z|<R\}$ для круга с центром в нуле радиуса $R$, $D=D_{1}$ для единичного круга. Пусть $H^{p}\left(D_{R}\right), 1 \leq p \leq \infty$, - пространство Харди функций $f$, аналитических в круге $D_{R}$, для которых конечна величина

$$
\begin{gathered}
\|f\|_{H^{p}\left(D_{R}\right)}=\sup _{\xi<R}\left(\int_{0}^{2 \pi}\left|f\left(\xi e^{i t}\right)\right|^{p} d t\right)^{1 / p} \quad \text { при } \quad 1 \leq p<\infty, \\
\|f\|_{H^{\infty}\left(D_{R}\right)}=\sup \left\{|f(z)|: z \in D_{R}\right\} \quad \text { при } \quad p=\infty .
\end{gathered}
$$

Пространство $H^{p}\left(D_{R}\right)$ наделено нормой, определяемой равенством (7.1). Введем классы Харди $Q_{p}\left(D_{R} ; N\right)$ и Харди - Соболева $Q_{r, p}^{n}\left(D_{R} ; N\right), N>0$, равенствами

$$
\begin{gathered}
Q_{p}\left(D_{R} ; N\right)=\left\{f \in H^{p}\left(D_{R}\right):\|f\|_{H^{p}\left(D_{R}\right)} \leq N\right\}, \\
Q_{r, p}^{n}\left(D_{R} ; N\right)=\left\{f \in H^{r}\left(D_{R}\right): f^{(n)} \in H^{p}\left(D_{R}\right),\left\|f^{(n)}\right\|_{H^{p}\left(D_{R}\right)} \leq N\right\} .
\end{gathered}
$$

Первое решение задачи Стечкина на классе аналитических функций было получено Л. В. Тайковым ([52], 1967). А именно, решение задачи (1.1) наилучшего приближения оператора дифференцирования порядка $k, 1 \leq k<n$, на классе $Q_{r, p}^{n}(D ; 1)$ множеством линейных 
ограниченных операторов $\mathcal{B}\left(H^{r}(D), H^{q}(D) ; N\right)$ из пространства Харди $H^{r}(D)$ в пространство Харди $H^{q}(D)$, норма которых не превосходит числа $N>0$, при условиях на параметры $r \geq 2$ и $1 \leq p, q \leq 2$. Также в работе [52] получено решение взаимосвязанной задачи наилучшего $H^{p}(D)$-приближения класса $Q_{p, r}^{k}(D ; 1)$ классом $Q_{p, q}^{n}(D ; N), N>0$. В статье [53] с использованием подхода из [52] результаты распространены на некоторый класс операторов и пространства Харди и Бергмана с весом.

В работе Л. В. Тайкова ([54], 1971) исследовалась задача наилучшего приближения по норме пространства $H^{p}\left(D_{\varrho}\right)$ класса Харди $Q_{p}\left(D_{\rho} ; 1\right)$ другим классом Харди $Q_{p}\left(D_{R} ; N\right)$ функций, аналитических в круге большего радиуса, при $\varrho<\rho<R$ и $1 \leq p \leq \infty$. Этой задаче приближения класса классом соответствует задача Стечкина на классе Харди $Q_{p}\left(D_{R} ; 1\right)$ наилучшего приближения оператора, сопоставляющего значениям функции $f \in H_{p}\left(D_{R}\right)$ на окружности $|z|=\varrho$ ее сужение на окружность $|z|=\rho$. Задача (3.2) состоит в данном случае в оптимальном восстановлении функции из $Q_{p}\left(D_{R} ; 1\right)$ на окружности $|z|=\rho$ по ее значениям на окружности $|z|=\varrho$, заданным с погрешностью. Эти задачи наилучшего приближения и оптимального восстановления оператора исследовались в [55]. Точные решения всех трех задач получены в случаях, когда теорема Адамара "о трех кругах" дает точное значение модуля непрерывности оператора.

Неравенства для норм производных колмогоровского типа в пространствах аналитических в круге функций рассматривали С.Б. Вакарчук, М.Б. Вакарчук, М.Ш. Шабозов, М. С. Саидусайнов и др. (см. ссылки в [53]).

В серии работ К. Ю. Осипенко и М. И. Стесина (см. [56-63] и ссылки там) изучались задачи оптимального восстановления аналитической в области функции и ее производных по приближенно заданным значениям на подмножестве области. Приведем некоторые результаты для классов функций, аналитических в полосе.

Пусть $H^{p}\left(S_{R}\right), 1 \leq p \leq \infty,-$ пространство аналитических в полосе $S_{R}=\{z \in \mathbb{C}:|\Im z|<R\}$ функций, для которых конечна величина

$$
\begin{gathered}
\|f\|_{H^{p}\left(S_{R}\right)}=\sup _{0<\xi<R}\left(\int_{-\infty}^{+\infty}|f(t-i \xi)|^{p} d t+\int_{-\infty}^{+\infty}|f(t+i \xi)|^{p} d t\right)^{1 / p} \text { при } 1 \leq p<\infty, \\
\|f\|_{H^{\infty}\left(S_{R}\right)}=\sup \left\{|f(z)|: z \in S_{R}\right\} \text { при } p=\infty .
\end{gathered}
$$

Пространство $H^{p}\left(S_{R}\right)$ наделено нормой, задаваемой равенством (7.2). Определим классы $Q_{p}\left(S_{R}\right)$ и $Q_{p}^{n}\left(S_{R}\right)$ равенствами

$$
\begin{gathered}
Q_{p}\left(S_{R}\right)=\left\{f \in H^{p}\left(S_{R}\right):\|f\|_{H^{p}\left(S_{R}\right)} \leq 1\right\} \\
Q_{p}^{n}\left(S_{R}\right)=\left\{f \in H^{p}\left(S_{R}\right): f^{(n)} \in H^{p}\left(S_{R}\right),\left\|f^{(n)}\right\|_{H^{p}\left(S_{R}\right)} \leq 1\right\} .
\end{gathered}
$$

В работах $[57 ; 58]$ решены задачи об оптимальном восстановлении первой и второй производной в точке на вещественной оси по следу функции на вещественной оси, заданному с погрешностью в равномерной норме, на классе $Q_{\infty}\left(S_{R}\right)$.

Отметим полученный К. Ю. Осипенко [59] аналог результата А. Н. Колмогорова. А именно, на классе $Q_{\infty}^{n}\left(S_{R}\right)$ вычислен модуль непрерывности оператора дифференцирования

$$
\omega(\delta)=\sup \left\{\left\|f^{(k)}\right\|_{L_{\infty}(\mathbb{R})}:\|f\|_{L_{\infty}(\mathbb{R})} \leq \delta,\left\|f^{(n)}\right\|_{H^{\infty}\left(S_{R}\right)} \leq 1\right\}
$$

при $1 \leq k \leq n+1, \delta \geq 0$ и $k=n+2, \delta \in\left(0, \delta_{n}\right]$, где $\delta_{n}$ - определенная величина.

В работе [62] получен аналог неравенства Харди - Литтлвуда - Полиа для функций, аналитических в полосе $S_{R}$. Точнее, на классе $Q_{2}^{n}\left(S_{R}\right)$ вычислен модуль непрерывности оператора дифференцирования порядка $k, 1 \leq k \leq n$,

$$
\omega(\delta)=\sup \left\{\left\|f^{(k)}\right\|_{L_{2}(\mathbb{R})}:\|f\|_{L_{2}(\mathbb{R})} \leq \delta,\left\|f^{(n)}\right\|_{H^{2}\left(S_{R}\right)} \leq 1\right\} .
$$


Решена взаимосвязанная задача оптимального восстановления в метрике $L_{2}(\mathbb{R})$ производной порядка $k$ по по неточно заданному следу функции на $\mathbb{R}$ на классе $Q_{2}^{n}\left(S_{R}\right)$.

В последние годы опубликован ряд работ Р. Р. Акопяна, посвященных задачам оптимального восстановления аналитической функции и ее производной по заданным с погрешностью предельным значениям на измеримой части границы области, на классе функций с ограниченной нормой на дополнительной части границы и взаимосвязанным задачам Стечкина (см. [55;64;65] и дальнейшие ссылки там). Все результаты получены с использованием взаимосвязи рассматриваемых задач, описанной в теореме 3. Остановимся подробно на двух из них.

Пусть $G$ - односвязная область комплексной плоскости, ограниченная кривой $\Gamma$, которая является жордановой спрямляемой кривой; $\gamma_{1}$ - измеримое подмножество $Г$ положительной меры и $\gamma_{0}$ - дополнение $\gamma_{1}$ до Г. Пусть $g(z, \zeta)$ - классическая функция Грина (задачи Дирихле) области $G$, а $\partial g / \partial n$ - производная функции Грина по внутренней для области $G$ нормали к кривой Г. Производную функции Грина по нормали также называют плотностью гармонической меры относительно области $G$ в точке $z$; в дальнейшем для нее будем использовать обозначение $P(z, \zeta)$. Соответственно гармоническая мера $w(z, \gamma, G)$ измеримого подмножества $\gamma$ спрямляемой границы $\Gamma$ относительно области $G$ в точке $z$ представима по формуле

$$
w(z, \gamma, G)=\int_{\gamma} P(z, \zeta)|d \zeta|
$$

Через $N_{*}(G)$ обозначим класс Смирнова (универсальный класс Харди) - класс аналитических в $G$ функций $f$, для которых гармоническая мажоранта функции $\ln ^{+}|f|$ существует и представима по формуле Грина. Функции класса $N_{*}(G)$ имеют почти всюду на $\Gamma$ некасательные (угловые) предельные граничные значения. Обозначаем функцию и ее граничные значения одинаково. Пусть $\varphi_{k}-$ неотрицательные измеримые весовые функции на $\gamma_{k}, k=0,1$; предполагаем суммируемость функций $\ln \varphi_{k}$ относительно плотности гармонической меры. Введем класс $\mathcal{H}=\mathcal{H}^{p, r}\left(G ; \gamma_{0}, \varphi_{0} ; \gamma_{1}, \varphi_{1}\right), p, r \geq 1$, аналитических в области $G$ функций из $N_{*}(G)$ таких, что их некасательные предельные граничные значения на $\gamma_{k}, k=0,1$, имеют

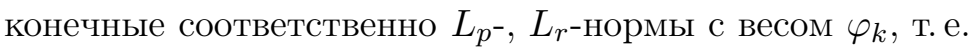

$\|f\|_{L_{p}\left(\gamma_{0}, \varphi_{0}\right)}=\left(\int_{\gamma_{0}}|f(\zeta)|^{p} \varphi_{0}(\zeta)|d \zeta|\right)^{1 / p}<+\infty, \quad\|f\|_{L_{r}\left(\gamma_{1}, \varphi_{1}\right)}=\left(\int_{\gamma_{1}}|f(\zeta)|^{r} \varphi_{1}(\zeta)|d \zeta|\right)^{1 / r}<+\infty$.

Если показатель нормы равен бесконечности, то конечна соответствующая $L_{\infty}$-норма:

$$
\|f\|_{L_{\infty}\left(\gamma_{k}\right)}=\operatorname{ess} \sup \left\{|f(\zeta)|: \zeta \in \gamma_{k}\right\} .
$$

В $\mathcal{H}$ выделим класс $Q=Q^{p, r}\left(G ; \gamma_{0}, \varphi_{0} ; \gamma_{1}, \varphi_{1}\right)$ функций $f$, которые удовлетворяют неравенству $\|f\|_{L_{p}\left(\gamma_{0}, \varphi_{0}\right)} \leq 1$.

В качестве приближаемого (восстанавливаемого) оператора $A$ рассматривается либо функционал $\Upsilon_{z_{0}}^{0}$, который ставит в соответствие граничным значениям на $\gamma_{1}$ функции $f$ ее значение в точке $z_{0}$ области $G$, либо $\Upsilon_{z_{0}}^{1}$, который сопоставляет граничным значениям на $\gamma_{1}$ функции $f$ ее производную $f^{\prime}\left(z_{0}\right)$. Формальные постановки задач таковы.

Для числа $\delta \geq 0$ и метода восстановления $T \in \mathcal{O}=\mathcal{O}\left(L_{r}\left(\gamma_{1}, \varphi_{1}\right), \mathbb{C}\right)$ величина

$$
\mathcal{U}(T, \delta)=\sup \left\{\left|f^{(s)}\left(z_{0}\right)-T g\right|: f \in Q, q \in L_{r}\left(\gamma_{1}, \varphi_{1}\right),\|f-q\|_{L_{r}\left(\gamma_{1}, \varphi_{1}\right)} \leq \delta\right\}
$$

является погрешностью восстановления значения в точке $z_{0}$ самой функции $(s=0)$ или ее производной $(s=1)$ для функций из класса $Q$ по граничным значениям функции на $\gamma_{1}$, заданным с погрешностью $\delta$ по норме $L_{r}\left(\gamma_{1}, \varphi_{1}\right)$, методом $T$. Тогда

$$
\mathcal{E}_{\mathcal{O}}(\delta)=\inf \{\mathcal{U}(T, \delta): T \in \mathcal{O}\}
$$


есть величина оптимального восстановления значения функции (производной) в точке $z_{0}$ (или, что то же самое, оптимального восстановления функционала $\left.\Upsilon_{z_{0}}^{s}\right)$ на классе $Q$ по приближенно заданным на $\gamma_{1}$ граничным значениям функции с помощью методов восстановления из множества $\mathcal{O}$. Задача состоит в вычислении величины $\mathcal{E}_{\mathcal{O}}(\delta)$ и определении оптимального метода восстановления - функционала, на котором в (7.3) достигается нижняя грань.

Задача вычисления значений аналитической функции по ее граничным значениям, заданным с погрешностью, является некорректной. Эта задача была исследована М. М. Лаврентьевым [66, гл. II, §1, пп. 4-5]. Для ее решения были предложены восстанавливающие операторы (называемые в теории некорректно поставленных задач методами регуляризации), которые имеют в качестве ядра функции, являющиеся по сути аппроксимациями ядра Коши, и названные функциями Карлемана. Цель задачи (7.3) - для каждых точки $z_{0} \in G$ и значения параметра $\delta$ построить метод восстановления, который являются наилучшим.

Величина

$$
U(T)=\sup \left\{\left|f^{(s)}\left(z_{0}\right)-T f\right|: f \in Q\right\}
$$

является уклонением функционала $T \in \mathcal{B}\left(L_{r}\left(\gamma_{1}, \varphi_{1}\right), \mathbb{C} ; N\right)$ от функционала $\Upsilon_{z_{0}}^{s}$ на классе функций $Q$. Соответственно величина

$$
E(N)=\inf \left\{U(T): T \in \mathcal{B}\left(L_{r}\left(\gamma_{1}, \varphi_{1}\right), \mathbb{C} ; N\right)\right\}
$$

есть наилучшее приближение функционала $\Upsilon_{z_{0}}^{s}$ множеством линейных ограниченных функционалов $\mathcal{B}\left(L_{r}\left(\gamma_{1}, \varphi_{1}\right), \mathbb{C} ; N\right)$ на классе $Q$. Задача состоит в вычислении величины $E(N)$ и построении экстремального функционала, на котором в (7.4) достигается нижняя грань.

В работе [64] получено решение задач для функционала $\Upsilon_{z_{0}}^{0}$, сопоставляющего предельным граничным значениям функции на $\gamma_{1}$ ее значение в точке $z_{0}$ односвязной области $G$.

По весовым функциям $\varphi_{k}, k=0,1$, для $\delta>0$ определим на границе $\Gamma$ области $G$ функцию $\psi_{\delta}$ по формуле

$$
\psi_{\delta}(\zeta)=\left\{\begin{array}{cl}
\left(\frac{P\left(z_{0}, \zeta\right)}{\beta \varphi_{0}(\zeta)}\right)^{1 / p}, \quad \zeta \in \gamma_{0}, \\
\delta\left(\frac{P\left(z_{0}, \zeta\right)}{\alpha \varphi_{1}(\zeta)}\right)^{1 / r}, \quad \zeta \in \gamma_{1},
\end{array}\right.
$$

в которой величины $\alpha=w\left(z_{0}, \gamma_{1}, G\right)$ и $\beta=1-\alpha=w\left(z_{0}, \gamma_{0}, G\right)$ соответственно равны гармонической мере $\gamma_{1}$ и $\gamma_{0}$ относительно области $G$ в точке $z_{0}$. Здесь и в дальнейшем, если $r$ и/или $p$ равны бесконечности, то считаем, что величины $1 / r$ и/или $1 / p$ соответственно равны нулю.

Определим функцию $s_{\delta} \in \mathcal{H}$ по функции $\psi_{\delta}$ равенством

$$
s_{\delta}(z)=\exp \left(u_{\delta}(z)+i v_{\delta}(z)\right), \quad z \in G,
$$

где функция

$$
u_{\delta}(z)=\int_{\Gamma} P(z, \zeta) \ln \psi_{\delta}(\zeta)|d \zeta|, \quad z \in G,
$$

является гармонической в области $G$, а $v_{\delta}$ - функция, гармонически сопряженная к $u_{\delta}$, единственная с точностью до вещественной аддитивной константы, выбор которой не важен.

На пространстве $L_{r}\left(\gamma_{1}, \varphi_{1}\right)$ определим линейный ограниченный функционал $T_{\delta}$ формулой

$$
T_{\delta} f=\int_{\gamma_{1}} P\left(z_{0}, \zeta\right) \frac{s_{\delta}\left(z_{0}\right)}{s_{\delta}(\zeta)} f(\zeta)|d \zeta|, \quad f \in L_{\varphi_{1}}^{q}\left(\gamma_{1}\right) .
$$

Далее будет использоваться величина $\mathcal{C}=\mathcal{C}^{p, r}\left(z_{0} ; \gamma_{0}, \varphi_{0} ; \gamma_{1}, \varphi_{1}\right)$, определяемая равенствами

$$
\begin{gathered}
\mathcal{C}=\left[\varepsilon\left(\gamma_{1}, \varphi_{1}\right)\right]^{1 / r}\left[\varepsilon\left(\gamma_{0}, \varphi_{0}\right)\right]^{1 / p} \alpha^{-\alpha / r} \beta^{-\beta / p}, \\
\varepsilon\left(\gamma_{k}, \varphi_{k}\right)=\exp \int_{\gamma_{k}} P\left(z_{0}, \zeta\right) \ln \frac{P\left(z_{0}, \zeta\right)}{\varphi_{k}(\zeta)}|d \zeta|, \quad k=0,1 .
\end{gathered}
$$


В работе [64] доказаны следующие утверждения.

Теорема 5. При произвольных $1 \leq r, p \leq \infty u \delta>0$ для величин оптимального восстановления (7.3) и модуля непрерывности функиионала $\Upsilon_{z_{0}}^{0}$ на классе $Q$ имеют место равенства

$$
\omega(\delta)=\mathcal{E}_{\mathcal{O}}(\delta)=\mathcal{C} \delta^{\alpha} .
$$

При этом экстремальными являются функиии вида $c s_{\delta},|c|=1$; в задаче (7.3) оптимальным методом восстановления является линейный ограниченный функиионал $T_{\delta}$.

Для функиий пространства $\mathcal{H}$ справедливо точное неравенство

$$
\left|f\left(z_{0}\right)\right| \leq \mathcal{C}\|f\|_{L_{r}\left(\gamma_{1}, \varphi_{1}\right)}^{\alpha}\|f\|_{L_{p}\left(\gamma_{0}, \varphi_{0}\right)}^{\beta} .
$$

Неравенство обращается в равенство на функи,ях вида $c s_{\delta}, \delta>0, c \in \mathbb{C}$.

Теорема 6. При произвольных $1 \leq r, p \leq \infty u N>0$ для величины наилучшего приближения (7.4) функиионала $\Upsilon_{z_{0}}^{0}$ на классе $Q$ справедливо равенство

$$
E(N)=\mathcal{C}^{1 / \beta} \beta \alpha^{\alpha / \beta} N^{-\alpha / \beta} .
$$

При этом в задаче (7.4) функиионалом наилучшего приближения является функиионал $T_{\delta}$, у которого параметр $\delta$ определен равенством $\delta=\mathcal{C}^{1 / \beta} \alpha^{1 / \beta} N^{-1 / \beta}$.

В [65] на классе $Q$ функций из пространства Харди $H^{\infty}(G)$, для которых $\|f\|_{L_{\infty}\left(\gamma_{0}\right)} \leq 1$, получено решение задач (7.3) и (7.4) при $p=r=\infty, \varphi_{0}=\varphi_{1} \equiv 1$ для функционала $\Upsilon_{z_{0}}^{1}$, который ставит в соответствие граничным значениям на $\gamma_{1}$ функции $f$ значение ее производной $f^{\prime}\left(z_{0}\right)$ в точке $z_{0}$ области $G$.

Обозначим через $w$ гармоническую в области $G$ функцию переменной $z$, значение которой в точке $z$ равно гармонической мере $\gamma_{1}$ относительно области $G$ в точке $z$, т. е. $w$ определена равенством $w(z)=w\left(z, \gamma_{1}, G\right)$. Будем использовать обозначения $\kappa\left(z_{0}\right), \bar{\nu}\left(z_{0}\right)$ и $t=t\left(z_{0}\right)$ соответственно для длины, направления и аргумента градиента $\nabla w\left(z_{0}\right)$ функции $w$ в точке $z_{0}$, определенных равенствами

$$
\kappa\left(z_{0}\right)=\left|\nabla w\left(z_{0}\right)\right|, \quad \bar{\nu}\left(z_{0}\right)=\frac{\nabla w\left(z_{0}\right)}{\left|\nabla w\left(z_{0}\right)\right|}, \quad \bar{\nu}\left(z_{0}\right)=(\cos t, \sin t) .
$$

Пусть $g$ - функция, задающая однолистное отображение области $G$ на единичный круг, удовлетворяющая условиям $g\left(z_{0}\right)=0, g^{\prime}\left(z_{0}\right)>0$. Обозначим через $\eta\left(z_{0}\right)$ положительную величину $\eta\left(z_{0}\right)=2 g^{\prime}\left(z_{0}\right) / \kappa\left(z_{0}\right)$, если $\kappa\left(z_{0}\right) \neq 0$, и равную $+\infty$, если $\kappa\left(z_{0}\right)=0$.

Пусть $s_{\delta}$ - функция, определяемая равенством (7.5) по функции $\psi_{\delta}$, определенной на границе $\Gamma$ формулой $\psi_{\delta}(\zeta)=\delta^{k}, \zeta \in \gamma_{k}, k=0,1$.

Для $\delta>0$ и точки $z_{0} \in G$, удовлетворяющих неравенству $|\ln \delta| \geq \eta\left(z_{0}\right)$, определим функционал $T_{\delta}^{1}$ на пространстве $L_{\infty}\left(\gamma_{1}\right)$ равенством

$$
T_{\delta}^{1} f=e^{-i t} \int_{\gamma_{1}} J_{z_{0}}(\zeta) \frac{s_{\delta}\left(z_{0}\right)}{s_{\delta}(\zeta)} f(\zeta)|d \zeta|,
$$

где

$$
J_{z_{0}}(\zeta)=\frac{\partial P}{\partial \bar{\nu}}\left(z_{0}, \zeta\right)+\ln \delta \kappa\left(z_{0}\right) P\left(z_{0}, \zeta\right) .
$$

Для $\delta>0$ и точки $z_{0} \in G$, удовлетворяющих неравенству $|\ln \delta|<\eta\left(z_{0}\right)$, определим в области $G$ функцию $F_{\delta}$ формулой

$$
F_{\delta}(z)=\frac{g(z)-g_{0}}{1-g(z) \overline{g_{0}}} s_{\delta}(z), \quad g_{0}=-e^{i t} \frac{\kappa\left(z_{0}\right) \ln \delta}{2 g^{\prime}\left(z_{0}\right)}=-e^{i t} \frac{\ln \delta}{\eta\left(z_{0}\right)} .
$$


В этом случае определим функционал $T_{\delta}^{1}$ равенством

$$
T_{\delta}^{1} f=e^{-i t} \int_{\gamma_{1}} I_{z_{0}}(\zeta) \frac{s_{\delta}\left(z_{0}\right)}{F_{\delta}(\zeta)} f(\zeta)|d \zeta|,
$$

в котором

$$
I_{z_{0}}(\zeta)=\frac{\ln \delta}{\eta\left(z_{0}\right)} \frac{\partial P}{\partial \bar{\nu}}\left(z_{0}, \zeta\right)+\kappa\left(z_{0}\right) \frac{1}{2}\left(\eta\left(z_{0}\right)+\frac{\ln ^{2} \delta}{\eta\left(z_{0}\right)}\right) P\left(z_{0}, \zeta\right)
$$

Следующие утверждения содержатся в [65].

Теорема 7. Для величин оптимального восстановления (7.3) и модуля непрерывности функиионала $\Upsilon_{z_{0}}^{1}$ при $p=r=\infty$ на классе $Q$ справедливы следующие утверждения.

(I) В случае $|\ln \delta| \geq \eta\left(z_{0}\right)$ справедливы равенства

$$
\omega(\delta)=\mathcal{E}_{\mathcal{O}}(\delta)=\kappa\left(z_{0}\right) \delta^{\alpha}|\ln \delta| .
$$

Экстремальными являются функиии вида $\mathrm{cs}_{\delta},|c|=1$, а оптимальным методом восстановления является линейный ограниченный функционал $T_{\delta}^{1}$, определенный равенством (7.6).

(II) В случае $|\ln \delta|<\eta\left(z_{0}\right)$ справедливы равенства

$$
\omega(\delta)=\mathcal{E}_{\mathcal{O}}(\delta)=\kappa\left(z_{0}\right) \delta^{\alpha} \frac{1}{2}\left(\eta\left(z_{0}\right)+\frac{\ln ^{2} \delta}{\eta\left(z_{0}\right)}\right) .
$$

Экстремальными являются функиии вида $c F_{\delta},|c|=1$, а оптимальным методом восстановления является линейный ограниченный функционал $T_{\delta}^{1}$, определенный равенством (7.7).

Теорема 8. В условиях теоремы 7 для величины наилучшего приближения функиионала $\Upsilon_{z_{0}}^{1}$ справедливъ следующие утверждения.

(I*) Если $N>0$ представимо в виде

$$
N=\kappa\left(z_{0}\right) \delta^{-\beta}|\alpha \ln \delta+1|, \quad|\ln \delta| \geq \eta\left(z_{0}\right),
$$

то справедливо равенство

$$
E(N)=\kappa\left(z_{0}\right) \delta^{\alpha}|\beta \ln \delta-1| .
$$

Функиионал $T_{\delta}^{1}$, определенный в (7.6), является функиионалом наилучшего приближения.

(II*) Если $N>0$ представимо в виде

$$
N=\kappa\left(z_{0}\right) \delta^{-\beta}\left[\frac{\alpha}{2}\left(\eta\left(z_{0}\right)+\frac{\ln ^{2} \delta}{\eta\left(z_{0}\right)}\right)+\frac{\ln \delta}{\eta\left(z_{0}\right)}\right], \quad|\ln \delta|<\eta\left(z_{0}\right)
$$

то справедливо равенство

$$
E(N)=\kappa\left(z_{0}\right) \delta^{\alpha}\left[\frac{\beta}{2}\left(\eta\left(z_{0}\right)+\frac{\ln ^{2} \delta}{\eta\left(z_{0}\right)}\right)-\frac{\ln \delta}{\eta\left(z_{0}\right)}\right] .
$$

Функиионал $T_{\delta}^{1}$, определенный в (7.7), является функиионалом наилучшего приближения.

Случаи $\left(\mathrm{I}^{*}\right)$ и $\left(\mathrm{II}^{*}\right)$ исчерпывают все возможные значения $N>0$.

Благодарность. Авторы признательны Е.Е. Бердышевой, которая тщательно прочитала рукопись статьи и сделала ряд весьма полезных замечаний. 


\section{СПИСОК ЛИТЕРАТУРЫ}

1. Стечкин С.Б. Неравенства между нормами производных произвольной функции // Acta Sci. Math. 1965. Vol. 26, № 3-4. P. 225-230.

2. Стечкин С.Б. Наилучшее приближение линейных операторов // Мат. заметки. 1967. Т. 1, вып. 2. C. $137-148$.

3. Стечкин С.Б. Избранные труды. Математика. М.: Наука. Физматлит, 1998. 384 с.

4. Иванов В.К., Васин В.В., Танана В.П. Теория линейных некорректных задач и ее приложения. М.: Наука, 1978. 206 с.

5. Арестов В.В., Габушин В.Н. Наилучшее приближение неограниченных операторов ограниченными // Изв. вузов. Мат. 1995. № 11. С. 42-68.

6. Арестов В.В. Приближение неограниченных операторов ограниченными и родственные экстремальные задачи // Успехи мат. наук. 1996. Т. 51, вып. 6. С. 89-124. doi: 10.4213/rm1019.

7. Тихомиров В.М., Магарил-Ильяев Г.Г. Неравенства для производных // Колмогоров А.Н. Избранные труды. Математика и механика. М.: Наука, 1985. С. 387-390.

8. Бабенко В.Ф., Корнейчук Н.П., Кофанов В.А., Пичугов С.А. Неравенства для производных и их приложения Киев: Наук. думка, 2003. 591 с.

9. Габушин В.Н. Наилучшее приближение функционалов на некоторых множествах // Мат. заметки. 1970. Т. 8, № 5. С. 551-562.

10. Hardy G.H., Littlewood J.E. Contribution to the arithmetic theory of series // Proc. London Math. Soc. (2). 1912. Vol. 11. P. 411-478.

11. Landau E. Einige Ungleichungen für zweimal differentierbare Funktionen // Proc. London Math. Soc. (2). 1913. Vol. 13. P. 43-49.

12. Hadamard J. Sur le module maximum d'une fonction et de ses dérivées // Soc. math. France, Comptes rendus des Séances. 1914. Vol. 41. P. 68-72.

13. Боссе Ю.Г. (Шилов Г.Е.) О неравенствах между производными // Сб. работ студ. науч. кружков МГУ. 1937. Т. 1. С. 17-27.

14. Колмогоров А.Н. О неравенствах между верхними гранями последовательных производных произвольной функции на бесконечном интервале // Избранные труды. Математика, механика. М.: Наука, 1985. С. 252-263. (Уч. зап. Моск. ун-та. Математика, кн. 3, 1939. Т. 30. С. 3-16.)

15. Габушин В.Н. Неравенства для норм функции и ее производных в метриках $L_{p} / /$ Мат. заметки. 1967. T. 1, № 3. C. 291-298.

16. Буслаев А.П., Магарил-Ильяев Г.Г., Тихомиров В.М. О существовании экстремальной функции в неравенстве для производных // Мат. заметки. 1982. Т. 32, № 6. С. 823-834.

17. Арестов В.В. О наилучшем приближении операторов дифференцирования // Мат. заметки. 1967. T. 1, № 2. C. 149-154.

18. Буслаев А.П. О приближении оператора дифференцирования // Мат. заметки. 1981. Т. 29, № 5. C. $731-742$.

19. Тимофеев В.Г. Метод Н. П. Купцова построения экстремальной функции в неравенстве между равномерными нормами производных функций на полуоси // Тр. Ин-та математики и механики УрО РAH. 2019. T. 25, № 2. C. 220-239. doi: 10.21538/0134-4889-2019-25-2-220-239 .

20. Субботин Ю.Н., Тайков Л.В. Наилучшее приближение оператора дифференцирования в пространстве $L_{2} / /$ Мат. заметки. 1968. Т. 3, № 2. С. 157-164.

21. Тайков Л.В. Неравенства типа Колмогорова и наилучшие формулы численного дифференцирования // Мат. заметки. 1968. Т. 4, № 2. С. 233-238.

22. Арестов В.В. О точных неравенствах между нормами функций и их производных // Acta Sci. Math. 1972. T. 33, № 3-4. P. 243-267.

23. Арестов В.В. Приближение операторов, инвариантных относительно сдвига // Тр. МИАН СССР. 1975. T. 138. C. $43-70$.

24. Арестов В.В. О наилучшем приближении операторов дифференцирования в равномерной метрике: дис. ... канд. физ.-мат. наук. Свердловск, 1969. 89 с.

25. Бердышев В.И. Наилучшее приближение в $L[0, \infty)$ оператора дифференцирования // Мат. заметки. 1971. Т. 9, № 5. С. 477-481.

26. Габушин В.Н. О наилучшем приближении оператора дифференцирования на полупрямой // Мат. заметки. 1969. Т. 6, № 5. С. 573-582.

27. Arestov V.V., Filatova M.A. Best approximation of the differentiation operator in the space $L_{2}$ on the semiaxis // J. Approx. Theory. 2014. Vol. 187. P. 65-81. doi: 10.1016/j.jat.2014.08.001. 
28. Харди Г.Г., Литтльвуд Дж.Е., Полиа Г. Неравенства. М.: ИЛ, 1948. 456 с.

29. Габушин В.Н. О наилучшем приближении оператора дифференцирования в метрике $L_{p} / /$ Мат. заметки. 1972. Т. 12, № 5. С. 531-538.

30. Арестов В.В. Наилучшее приближение неограниченных операторов, инвариантных относительно сдвига, линейными ограниченными операторами // Тр. МИАН. 1992. Т. 198. С. 3-20.

31. Арестов В.В. О равномерной регуляризации задачи вычисления значений оператора // Мат. заметки. 1977. Т. 22, № 2. С. 231-244.

32. Арестов В.В. Наилучшее восстановление операторов и родственные задачи // Тр. МИАН СССР. 1989. T. 189. С. $3-20$.

33. Стейн И., Вейс Г. Введение в гармонический анализ на евклидовых пространствах. М.: Мир, 1974. 333 c.

34. Lorentz G.G. Approximation of functions. N Y: Holt, Rinehalt \& Winston, 1966. 188 p.

35. Тихомиров В.М. Некоторые вопросы теории приближений. М., Изд-во МГУ, 1976. 304 с.

36. Арестов В.В. Приближение инвариантных операторов // Мат. заметки. 1983. Т. 34, № 1. С. 9-29.

37. Арестов В.В. Приближение операторов типа сверки линейными ограниченными операторами // Тр. МИАН. 1980. Т. 145. С. 3-19.

38. Арестов В.В. О наилучшем приближении оператора дифференцирования // Приближение функций полиномами и сплайнами: сб. ст. Свердловск, 1985. С. 3-14.

39. Arestov V.V. On the best approximation of the differentiation operator // Ural Math. J. 2015. Vol. 1, no. 1. P. 20-29. doi: 10.15826/umj.2015.1.002 .

40. Хермандер Л. Оценки для операторов, инвариантных относительно сдвига. М.: ИЛ, 1962. 71 с.

41. Larsen R. An introduction to the theory of multipliers. Berlin etc.: Springer, 1971. 282 p.

42. Figà-Talamanca A. Translation invariant operators in $L^{p} / /$ Duke. Math. J. 1965. Vol. 32. P. 495-502.

43. Арестов В.В. О сопряженности пространства мультипликаторов // Тр. Ин-та математики и механики УрО РАН. 2019. Т. 25, № 4. С. 5-14. doi: 10.21538/0134-4889-2019-25-4-5-14 .

44. Arestov V. Uniform approximation of differentiation operators by bounded linear operators in the space $L_{r} / /$ Anal. Math. 2020. Vol. 46, no. 3. P. 425-445. doi: 10.1007/s10476-020-0040-z .

45. Арестов В.В. Наилучшее равномерное приближение оператора дифференцирования ограниченными в пространстве $L_{2}$ операторами // Тр. Ин-та математики и механики УрО РАН. 2018. Т. 24, № 4. C. 34-56. doi: 10.21538/0134-4889-2018-24-4-34-56.

46. Arestov V.V. Best approximation of a differentiation operator on the set of smooth functions with exactly or approximately given Fourier transform // Mathematical Optimization Theory and Operations Research (MOTOR 2019) / eds. M. Khachay, Y. Kochetov, P. Pardalos. Cham: Springer, 2019. P. 434-448.

47. Тиман А.Ф. Теория приближения функций действительного переменного. М.: Физматгиз, 1960. $624 \mathrm{c}$.

48. Коновалов В.Н. Точные неравенства для норм функций, третьих частных, вторых смешанных или косых производных // Мат. заметки. 1978. Т. 23, № 1. С. 67-78.

49. Тимошин О.А. Наилучшее приближение оператора второй смешанной производной в метриках $L$ и $C$ на плоскости // Мат. заметки. 1984. Т. 36, № 3. С. 369-375.

50. Тимошин О.А. Точные неравенства между нормами частных производных второго и третьего порядка // Докл. РАН. 1995. Т. 344, № 1. С. 20-22.

51. Тимофеев В.Г. Неравенство типа Ландау для функций нескольких переменных // Мат. заметки. 1985. Т. 37 , № 5. С. $676-689$.

52. Тайков Л.В. О наилучшем приближении в среднем некоторых классов аналитических функций // Мат. заметки. 1967. Т. 1, вып. 2. С. 155-162.

53. Акопян P.P., Саидусайнов М.C. Три экстремальные задачи в пространствах Харди и Бергмана аналитических функций в круге // Тр. Ин-та математики и механики УрО РАН. 2017. Т. 23, № 3. C. $22-32$.

54. Тайков Л.В. Аналитическое продолжение функций с ошибкой // Тр. МИАН СССР. 1971. Т. 109. C. $61-64$.

55. Акопян P.P. Наилучшее приближение оператора аналитического продолжения на классе аналитических в кольце функций // Тр. Ин-та математики и механики УрО РАН. 2012. Т. 18, № 4. C. $3-13$.

56. Осипенко К.Ю., Стесин М.И. О некоторых задачах оптимального восстановления аналитических и гармонических функций по неточным данным // Сиб. мат. журн. 1993. Т. 34, № 3. C. $144-160$. 
57. Осипенко К.Ю., Стесин М.И. Оптимальное восстановление производных ограниченных аналитических и гармонических функций по неточным данным // Мат. заметки. 1993. Т. 53, № 5. C. 87-97.

58. Осипенко К.Ю. Об $n$-поперечниках, оптимальных квадратурных формулах и оптимальном восстановлении функций, аналитических в полосе // Изв. РАН. Сер. математическая. 1994. Т. 58, № 4. C. $55-79$.

59. Осипенко К.Ю. Неравенства для производных аналитических в полосе функций // Мат. заметки. 1994. T. 56, № 4. C. 114-122.

60. Osipenko K.Yu. Optimal recovery of analytic functions. Huntington: NOVA Science Publ.Inc., 2000. $229 \mathrm{p}$.

61. Осипенко К.Ю. Об оптимальных методах восстановления в пространствах Харди - Соболева / / Мат. сб. 2001. Т. 192, № 2. С. 67-86.

62. Осипенко К.Ю. Неравенство Харди - Литтлвуда - Полиа для аналитических функций из пространств Харди - Соболева // Мат. сб. 2006. Т. 197, № 3. С. 15-34.

63. Osipenko K.Yu., Stessin M.I. Hadamard and Schwarz type theorems and optimal recovery in spaces of analytic functions // Constr. Approx. 2010. Vol. 31. P. 37-67. doi: 10.1007/s00365-009-9043-5 .

64. Акопян P.P. Аналог теоремы о двух константах и оптимальное восстановление аналитических функций // Мат. сб. 2019. Т. 210, № 10. С. 3-36.

65. Akopyan R.R. Optimal recovery of a derivative of an analytic function from values of the function given with an error on a part of the boundary. II // Anal. Math. 2020. Vol. 46, № 3. P. 409-424. doi: 10.1007/s10476-020-0039-5.

66. Лаврентьев М.М., Романов В.Г., Шишатский С.П. Некорректные задачи математической физики и анализа. 1980. М.: Наука. 286 с.

Поступила 11.10.2020

После доработки 1.11.2020

Принята к публикации 16.11.2020

Арестов Виталий Владимирович

доктор физ.-мат. наук, профессор

Уральский федеральный университет;

ведущий научный сотрудник

Институт математики и механики им. Н.Н. Красовского УрО РАН

г. Екатеринбург

e-mail: vitalii.arestov@urfu.ru

Акопян Роман Размикович

канд. физ.-мат. наук, доцент

Уральский федеральный университет;

Институт математики и механики им. Н.Н. Красовского УрО РАН

г. Екатеринбург

e-mail: RRAkopyan@mephi.ru

\section{REFERENCES}

1. Stechkin S.B. Inequalities between norms of derivatives of arbitrary functions. Acta Sci. Math., 1965, vol. 26, no. 3-4, pp. 225-230 (in Russian).

2. Stechkin S.B. Best approximation of linear operators. Math. Notes, 1967, vol. 1, no. 2, pp. 91-99. doi: $10.1007 / \mathrm{BF} 01268056$.

3. Stechkin S.B. Izbrannye trudy: Matematika (Selected Works: Mathematics). Moscow: Nauka Publ., 1998, 384 pp. ISBN: 5-02-015239-0.

4. Ivanov V.K., Vasin V.V., Tanana V.P. Theory of linear Ill-posed problems and its applications. Utrecht: VSP, 2002, 294 p. ISBN: 3111826147. Original Russian text published in Ivanov V.K., Vasin V.V., Tanana V.P. Teorija linejnyh nekorrektnyh zadach i ee prilozhenija. Moscow: Nauka Publ., 1978, 206 p. 
5. Arestov V.V., Gabushin V.N. Best approximation of unbounded operators by bounded operators. Russian Math. (Iz. VUZ), 1995, vol. 39, no. 11, pp. 38-63.

6. Arestov V.V. Approximation of unbounded operators by bounded operators and related extremal problems, Russian Math. Surveys, 1996, vol. 51, no. 6, pp. 1093-1126. doi: 10.1070/RM1996v051n06ABEH003001.

7. Tikhomirov V.M., Magaril-Il'yaev G.G. Neravenstava dlya proizvodnykh [Inequalirties for derivatives]. In: Kolmogorov A.N. Selected Works: Mathematics and Mechanics. Moscow: Nauka Publ., 1985, pp. 387390 (in Russian).

8. Babenko V.F., Korneichuk N.P., Kofanov V.A. and Pichugov S.A. Neravenstva dlya proizvodnykh i ikh prilozheniya [Inequalities for derivatives and their applications]. Kiev: Naukova Dumka, 2003, 590 p. ISBN: 966-00-0074-4.

9. Gabushin V.N. Best approximations of functionals on certain sets. Math. Notes, 1970, vol. 8, no. 5, pp. 780-785. doi: 10.1007/BF01146932 .

10. Hardy G.H., Littlewood J.E. Contribution to the arithmetic theory of series // Proc. London Math. Soc. (2). 1912. Vol. 11. P. 411-478.

11. Landau E. Einige Ungleichungen für zweimal differentierbare Funktionen. Proc. London Math. Soc. (2), 1913, vol. 13, pp. 43-49. doi: 10.1112/plms/s2-13.1.43.

12. Hadamard J. Sur le module maximum d'une fonction et de ses dérivées. Soc. math. France, Comptes rendus des Séances, 1914, vol. 41, pp. 68-72.

13. Bosse Yu.G. (Shilov G.E.) On inequalities between derivatives. In: Collection of Works of Student Scientific Societies of Moscow State University, vol. 1. Moscow: Moscow University, 1937, pp. 17-27 (in Russian).

14. Kolmogorov A.N. On inequalities between upper bounds of consecutive derivatives of an arbitrary function defined on an infinite interval. In: Selected Works. Mathematics and Mechanics. Moscow: Nauka Publ., 1985, pp. 252-263; Moskov. Gos. Univ., Uchenye Zap. (Mat. 3), 1939, vol. 30, pp. 3-16 (in Russian).

15. Gabushin, V.N. Inequalities for the norms of a function and its derivatives in metric $L_{p}$. Math. Notes, 1967, vol. 1, pp. 194-198. doi: 10.1007/BF01098882.

16. Buslaev A.P., Magaril-Il'yaev G.G., Tikhomirov V.M., Existence of extremal functions in inequalities for derivatives. Math. Notes, 1982, vol. 32, no. 6, pp. 898-904. doi: 10.1007/BF01145874.

17. Arestov V.V. On the best approximation of differentiation operators. Math. Notes, 1967, vol. 1, no. 2, pp. 100-103. doi: 10.1007/BF01268057.

18. Buslaev A.P. Approximation of a differentiation operator, Math. Notes, 1981, vol. 29, no. 5, pp. 372-378. doi: $10.1007 / \mathrm{BF} 01158361$.

19. Timofeev V.G. N.P.Kuptsov's method for the construction of an extremal function in an inequality between uniform norms of derivatives of functions on the half-line, Tr. Inst. Mat. Mekh. UrO RAN, 2019, vol. 25, no. 2, pp. 220-239 (in Russian). doi: 10.21538/0134-4889-2019-25-2-220-239 .

20. Subbotin Yu.N., Taikov L.V. Best approximation of a differentiation operator in $L_{2}$-space. Math. Notes, 1968, vol. 3, no. 2, pp. 100-105. doi: 10.1007/BF01094328.

21. Taikov L.V. Kolmogorov-type inequalities and the best formulas for numerical differentiation. Math. Notes, 1968, vol. 4, pp. 631-634. doi: 10.1007/BF01094964.

22. Arestov V.V. On best inequalities between norms of functions and their derivatives. Acta Sci. Math., 1972, vol. 33, pp. 243-267.

23. Arestov V.V. Approximation of operators invariant with respect to a shift. Proc. Steklov Inst. Math., 1975, vol. 138, pp. 45-74.

24. Arestov V.V. O nailuchshem priblizhenii operatorov differentsirovaniya $v$ ravnomernoi metrike [On the best approximation of differentiation operators in the uniform metric]. Dissertation, Cand. Sci. (Phys.-Math.), Sverdlovsk, 1969, 89 p.

25. Berdyshev V.I. Best approximations in $L[0$, infty $)$ of the differentiation operator. Math. Notes, 1971, vol. 9, no. 5, pp. 275-277. doi: 10.1007/BF01094351.

26. Gabushin V.N. On the best approximation of the differentiation operator on the half-line. Math. Notes, 1969, vol. 6, pp. 804-810. doi: 10.1007/BF01101408.

27. Arestov V.V., Filatova M.A. Best approximation of the differentiation operator in the space $L_{2}$ on the semiaxis. J. Approx. Theory, 2014, vol. 187, pp. 65-81. doi: 10.1016/j.jat.2014.08.001 .

28. Hardy G.H., Littlewood J.E., Pólya G. Inequalities. Cambridge: Cambridge University Press, 1934, 340 p. ISBN(2nd ed.): 0-521-05206-8. Translated to Russian under the title Neravenstva. Moscow: Inostr. Lit. Publ., 1948, 456 p. 
29. Gabushin V.N. The best approximation of the differentiation operator in the metric of $L_{p}$. Math. Notes, 1972, vol. 12, no. 5, pp. 756-760. doi: 10.1007/BF01099059 .

30. Arestov V.V. Best approximation of translation invariant unbounded operators by bounded linear operators. Proc. Steklov. Inst. Math., 1994, vol. 198, pp. 1-16.

31. Arestov V.V. Uniform regularization of the problem of calculating the values of an operator. Math. Notes, 1977, vol. 22, pp. 618-626. doi: 10.1007/BF01780971.

32. Arestov V. V. Optimal recovery of operators and related problems. Trudy Mat. Inst. Steklov, 1989, vol. 189, pp. 3-20.

33. Stein E.M., Weiss G. Introduction to Fourier analysis on Euclidean spaces. Princeton: Princeton Univ. Press, 1971, 312 p. ISBN: 9781400883899. Translated to Russian under the title Vvedenie v garmonicheskii analiz na evklidovykh prostranstvakh. Moscow: Mir Publ., 1974, 338 p.

34. Lorentz G.G. Approximation of functions. N Y: Holt, Rinehalt \& Winston, 1966, 188 p. ISBN: 9780030552205 .

35. Tikhomirov V.M. Nekotorye voprosy teorii priblizhenii [Some questions of approximation theory]. Moscow: Izd. Mosk. Gos. Univ., 1976, 304 p.

36. Arestov V.V. Approximation of invariant operators. Math. Notes, 1983, vol. 34, no. 1, pp. $489-499$. doi: 10.1007/BF01160861 .

37. Arestov V.V. Approximation of operators of convolution type by bounded linear operators. Proc. Steklov Inst. Math., 1981, vol. 145, pp. 1-18.

38. Arestov V.V. On the best approximation of the differentiation operator. In: Priblizhenie funktsii polinomami $i$ splainami [Approximation of functions by polynomials and splines, Collected papers]. Sverdlovsk, 1985, pp. 3-14.

39. Arestov V.V. On the best approximation of the differentiation operator. Ural Math. J., 2015, vol. 1, no. 1, pp. 20-29. doi: 10.15826/umj.2015.1.002 .

40. Hörmander L. Estimates for translation invariant operators in $l^{p}$ spaces. Acta Mathematica, 1960, vol. 104, pp. 93-140. doi: 10.1007/BF02547187.

41. Larsen R. An introduction to the theory of multipliers. Berlin etc.: Springer, 1971, 282 p. doi: 10.1007/9783-642-65030-7.

42. Figà-Talamanca A. Translation invariant operators in $L^{p}$. Duke. Math. J., 1965, vol. 32, pp. $495-502$. doi: 10.1215/S0012-7094-65-03250-3 .

43. Arestov V.V. On the conjugacy of the space of multipliers. Tr. Inst. Mat. Mekh. UrO RAN, 2019, vol. 25, no. 4, pp. 5-14 (in Russian). doi: 10.21538/0134-4889-2019-25-4-5-14.

44. Arestov V. Uniform approximation of differentiation operators by bounded linear operators in the space $L_{r}$. Anal. Math., 2020, vol. 46, no. 3, pp. 425-445. doi: 10.1007/s10476-020-0040-z .

45. Arestov V.V. Best uniform approximation of the differentiation operator by operators bounded in the space $L_{2}$. Proc. Steklov Inst. Math., 2020, vol. 308, Suppl. 1, pp. S9-S30. doi: 10.1134/S0081543820020029.

46. Arestov V.V. Best approximation of a differentiation operator on the set of smooth functions with exactly or approximately given Fourier transform. In: Khachay M., Kochetov Y., Pardalos P. (eds) Mathematical Optimization Theory and Operations Research (MOTOR 2019), Lecture Notes in Computer Science, vol. 11548. Cham: Springer, 2019, pp. 434-448. doi: 10.1007/978-3-030-22629-9_30.

47. Timan A.F. Theory of approximation of functions of a real variable. N Y, Pergamon, 1963. Original Russian text published in Teoriya priblizheniya funktsii deistvitel'nogo peremennogo, Moscow, Fizmatgiz, 1960, $624 \mathrm{p}$.

48. Konovalov V.N. Precise inequalities for norms of functions, third partial, second mixed, or directional derivatives. Math. Notes, 1978, vol. 23, pp. 38-44. doi: 10.1007/BF01104884.

49. Timoshin O.A. Best approximation of the operator of second mixed derivative in the metrics of $L$ and $C$ on the plane. Math. Notes, 1984, vol. 36, pp. 683-686. doi: $10.1007 /$ BF01141940.

50. Timoshin O.A. Sharp inequalities between norms of partial derivatives of second and third order. Dokl. Akad. Nauk, 1995, vol. 344, no. 1, pp. 20-22 (in Russian).

51. Timofeev V.G. Landau inequality for function of several variables. Math. Notes, 1985, vol. 37, pp. 369-377. doi: 10.1007/BF01157968 .

52. Taikov L. V. Best approximation in the mean of certain classes of analytic functions. Math. Notes, 1967, vol. 1, no. 2, pp. 104-109. doi: 10.1007/BF01268058.

53. Akopyan R.R., Saidusainov M.S. Three extremal problems in the Hardy and Bergman spaces of functions analytic in a disk. Proc. Steklov Inst. Math., 2018, vol. 303, suppl. 1, pp. S25-S35. doi: $10.1134 /$ S0081543818090031 . 
54. Taikov L.V. Analytic continuation of functions with an error. Proc. Steklov Inst. Math., 1971, vol. 109, pp. $68-72$.

55. Akopyan R.R. Best approximation for the analytic continuation operator on the class of analytic functions in a ring. Trudy Inst. Mat. Mekh. UrO RAN, 2012, vol. 18, no. 4, pp. 3-13 (in Russian).

56. Osipenko K. Yu., Stesin M. I. On some problems of optimal recovery of analytic and harmonic functions from inaccurate data. Siberian Math. J., 1993, vol. 34, no. 3, pp. 523-539. doi: 10.1007/BF00971228.

57. Osipenko K.Yu., Stesin M.I. Optimal recovery of derivatives of bounded analytic and harmonic functions from inaccurate data. Math. Notes, 1993, vol. 53, no 5, pp. 513-520. doi: 10.1007/BF01208547.

58. Osipenko K.Yu. On $n$-widths, optimal quadrature formulas, and optimal recovery of functions analytic in a strip. Russ. Acad. Sci. Izv. Math., 1995, vol. 45, no 1, pp. 55-78. doi: 10.1070/IM1995v045n01ABEH001635 .

59. Osipenko K.Yu. Inequalities for derivatives of functions analytical in a strip. Math. Notes, 1994, vol. 56, no. 4, pp. 1069-1074. doi: 10.1007/BF02362376.

60. Osipenko K.Yu. Optimal recovery of analytic functions. Huntington: NOVA Science Publ. Inc., 2000, 229 p. ISBN: 1-56072-821-3.

61. Osipenko K.Yu. On optimal recovery methods in Hardy-Sobolev spaces. Sb. Math., 2001, vol. 192, no. 2, pp. 225-244. doi: 10.1070/SM2001v192n02ABEH000543.

62. Osipenko K.Yu. The Hardy-Littlewood-Porlya inequality for analytic functions in Hardy-Sobolev spaces. Sb. Math., 2006, vol. 197, no. 3, pp. 315-334. doi: 10.1070/SM2006v197n03ABEH003760.

63. Osipenko K.Yu., Stessin M.I. Hadamard and Schwarz type theorems and optimal recovery in spaces of analytic functions. Constr. Approx., 2010, vol. 31, pp. 37-67. doi: 10.1007/s00365-009-9043-5 .

64. Akopyan R.R. An analogue of the two-constants theorem and optimal recovery of analytic functions. Sb. Math., 2019, vol. 210, no. 10, pp. 1348-1360. doi: 10.1070/SM8952.

65. Akopyan R.R. Optimal recovery of a derivative of an analytic function from values of the function given with an error on a part of the boundary. II. Anal. Math., 2020, vol. 46, no 3, pp. 409-424. doi: 10.1007/s10476-020-0039-5 .

66. Lavrent'ev M.M., Romanov V.G., Shishatskii S.P. Ill-posed problems of mathematical physics and analysis. Transl. Math. Monogr., vol. 64, Providence, RI: Amer. Math. Soc., 1986, 290 pp. ISBN: 0821898140 . Original Russian text published in Lavrent'ev M.M., Romanov V.G., Shishatskii S.P. Nekorrektnye zadachi matematicheskoi fiziki i analiza. Moscow: Nauka Publ., 1980, 286 p.

Received October 11, 2020

Revised November 1, 2020

Accepted November 16, 2020

Funding Agency: This work was performed as a part of the research conducted in the Ural Mathematical Center and also supported by the Russian Foundation for Basic Research (project no. 18-01-00336) and by the Russian Academic Excellence Project (agreement no. 02.A03.21.0006 of August 27, 2013, between the Ministry of Education and Science of the Russian Federation and Ural Federal University).

Vitalii Vladimirovich Arestov, Dr. Phys.-Math. Sci., Ural Federal University, Yekaterinburg, 620000 Russia; Krasovskii Institute of Mathematics and Mechanics of the Ural Branch of the Russian Academy of Sciences, Yekaterinburg, 620108 Russia; e-mail: vitalii.arestov@urfu.ru .

Roman Razmikovich Akopyan, Cand. Sci. (Phys.-Math.), Ural Federal University, Yekaterinburg, 620000 Russia; Krasovskii Institute of Mathematics and Mechanics of the Ural Branch of the Russian Academy of Sciences, Yekaterinburg, 620108 Russia; e-mail: RRAkopyan@mephi.ru .

Cite this article as: V.V. Arestov, R. R. Akopyan. Stechkin's problem on the best approximation of an unbounded operator by bounded ones and related problems, Trudy Instituta Matematiki $i$ Mekhaniki UrO RAN, 2020, vol. 26, no. 4, pp. 7-31. 